\title{
Moneyspots
}

\section{Extraneous Attributes and the Coexistence of Money and Interest-Bearing Nominal Bonds}

\author{
Ricardo Lagos* \\ New York University
}

February 2010

\begin{abstract}
It is folklore among monetary theorists that, under laissez faire, absent ad-hoc assumptions that favor money over bonds, there do not exist equilibria in which government-issued fiat money coexists with nominal risk-free, interest-bearing government bonds with similar physical characteristics. This proposition is the basis for the strongest version of the rateof-return-dominance puzzle. In this paper I show that if - as has been the case throughout monetary history - the physical object used as fiat money is heterogeneous in an extraneous attribute, then there exist equilibria in which money coexists with interest-bearing bonds.
\end{abstract}

Keywords: Money, nominal bonds, rate-of-return dominance JEL Classification: E41, E43

*I thank David Andolfatto, Huberto Ennis, Daniela Puzzello, and Neil Wallace for their feedback. Financial support from the C.V. Starr Center for Applied Economics at NYU is gratefully acknowledged. 


\begin{abstract}
"What has to be explained is the decision to hold assets in the form of barren money, rather than of interest- or profit-yielding securities.[...] This, as I see it, is really the central issue in the pure theory of money. Either we have to give an explanation of the fact that people do hold money when rates of interest are positive, or we have to evade the difficulty somehow."
\end{abstract}

John Hicks, 1935.

\title{
1 Introduction
}

At least since Hicks (1935), the basic observation that in actual economies money coexists with securities that bear a higher financial return, has been regarded as the central challenge facing monetary theory. Because this observation has proven difficult to rationalize in economic models, it has come to be known as the rate-of-return-dominance puzzle (or coexistence puzzle). The rate-of-return-dominance puzzle is one of the two fundamental questions that constitute the core of monetary theory. The other, more elementary question, is that of existence of a monetary equilibrium as a way to rationalize the fact that fiat money sells at a positive price for valuable goods and services in actual economies, despite it being intrinsically useless, and a formal claim to nothing, against no one. Much has been learned about the existence question by building models that explicitly incorporate frictions (e.g., limited commitment and enforcement, decentralized exchange, double-coincidence-of-wants problems) and identify conditions that can make monetary exchange an equilibrium. In contrast, the issue of rate-of-return-dominance has received scant attention.

One reason why monetary economists may have been disinclined to tackle the rate-of-returndominance puzzle is that the stronger versions of the puzzle, i.e., those in which the security that dominates money in rate of return is very similar to money, appear unsolvable: It is folklore among monetary theorists that, under laissez faire, absent ad-hoc assumptions that favor money over bonds, there do not exist equilibria in which government-issued fiat money coexists with nominal risk-free, interest-bearing, payable-to-the-bearer government bonds with similar physical characteristics. Because there have been instances in which such securities were issued and money remained in circulation, this folk impossibility proposition is the basis for the strongest version of the rate-of-return-dominance puzzle.

In this paper I show that if the physical object used as fiat money is heterogeneous in some extraneous attribute - a moneyspot such as a serial number - then there exist equilibria 
in which money coexists with interest-bearing bonds. ${ }^{1}$ I provide an explicit characterization of a class of equilibria that can rationalize the rate-of-return-dominance puzzle in its strongest form, and show that these equilibria can exhibit "liquidity effects" of open-market operations that resemble those found in limited-participation models, but without actually assuming that participation in asset markets is limited. I also show that moneyspots can overturn another folk proposition, i.e., that it is impossible for the nominal interest rate on government bonds to ever become negative if private agents can costlessly store money.

The rest of the paper is organized as follows. Section 2 presents the model. Section 3 considers the special case of no moneyspots, and formulates a version of the folk impossibility proposition. Section 4 analyzes the general case with moneyspots, and presents the coexistence proposition. Section 5 discusses additional theoretical implications of moneyspots for the behavior of the nominal interest rate, such as the liquidity effects often associated with open-market sales, and the possibility that nominal interest rates become negative. Section 6 concludes with a discussion of the findings. Appendix A contains the proofs of the main propositions, Appendix B contains subsidiary proofs, and Appendix C reviews the related literature.

\section{The model}

The model builds on Lagos and Wright (2005). Time is represented by a sequence of periods indexed by $t=0,1, \ldots$ Each time-period is divided into two subperiods where different activities take place. There is a continuum of infinitely lived agents, each identified with a point in the set $\mathcal{I}=[0,1]$. There are two nonstorable and perfectly divisible consumption goods at each date: general goods, and special goods.

In each subperiod, every agent is endowed with $\bar{h}$ units of time which can be employed as labor services. In the second subperiod, each agent has access to a linear production technology that transforms labor services into general goods. General goods are homogeneous, and consumed by all agents. Special goods instead come in many varieties. In the first subperiod, each agent has access to a linear production technology that transforms his own labor input into a

\footnotetext{
${ }^{1}$ Throughout this paper, "moneyspots" is used to represent extraneous attributes of fiat money, e.g., the serial numbers on notes, the mint years stamped on coins, the markings that identify the region where the issuing mint is located, the degree of wear and tear, etc. The term is intended to be reminiscent of "sunspots" (Cass and Shell, 1983). Sunspots, the realization of a publicly observed extrinsic random variable, can sometimes be used to coordinate actions in a way that generates self-fulfilling prophecies and enlarges the set of equilibria. I will show that, by introducing the possibility of symmetry-breaking self-fulfilling prophecies, moneyspots can enlarge the set of monetary equilibria. This observation will be the basis for the coexistence result.
} 
particular variety of the special good that he himself does not consume. This specialization is formalized as follows. Given two agents $i$ and $j$ drawn at random, the probability that $i$ consumes the variety of special good that $j$ produces but not vice-versa (a single coincidence) is denoted $\alpha$, with $\alpha \leq 1 / 2$. The probability that $j$ consumes the special good that $i$ produces but not vice-versa is also $\alpha$. The probability neither $i$ nor $j$ wants what the other agent can produce is $1-2 \alpha$. In a single-coincidence meeting, the agent who wishes to consume is the buyer, and the agent who produces, the seller. Let $\mathcal{I}_{t}^{b} \subseteq \mathcal{I}$ and $\mathcal{I}_{t}^{s} \subseteq \mathcal{I}$ denote the subsets of agents that, in the decentralized market of period $t$, act as buyers and sellers, respectively.

In the first subperiod, agents participate in a decentralized market where trade is bilateral (each meeting is a random draw from the set of pairwise meetings), and the terms of trade are determined by bargaining consisting of a take-it-or-leave-it offer by the buyer. The specialization of agents over consumption and production of the special good combined with bilateral trade, gives rise to a double-coincidence-of-wants problem. In the second subperiod, agents trade in a competitive centralized market. Agents cannot make binding commitments, and histories of actions are private in a way that precludes any borrowing and lending, so all trade - both in centralized and decentralized markets - is quid pro quo.

There is a monetary authority or "government" that can issue a financial security called money. Each unit of money is physically represented by a note - a piece of paper which is durable, uncounterfeitable, and intrinsically useless (the note itself is not an argument of any utility or production function). The initial stock of notes outstanding at the beginning of period 0 is represented by the set $\mathcal{M}_{0}=\left[0, M_{0}\right]$, where $M_{0} \in \mathbb{R}^{+}$is given. Every note is physically identical to every other note (e.g., equal in size, color, denomination, and other markings), except for the fact that each note is uniquely identified by an extraneous attribute or moneyspot: a serial number $n \in \mathcal{M}_{0}$ which is printed on it. ${ }^{2}$ The government can also issue nominal one-period bearer default-free pure discount bonds. Each bond is represented by a piece of paper which is durable, uncounterfeitable, and intrinsically useless. A bond issued in

\footnotetext{
${ }^{2}$ François Velde has pointed out to me that the practice of identifying notes with serial numbers is very old, e.g., the edict creating John Law's bank in 1716 specifies in its annex the form that the notes to the bearer should take, and they were numbered. The serial number is also one of the most universal features of paper money. It is difficult to find a class of notes that has ever circulated where each note was not uniquely identified by a serial number (some rare cases have been recorded, e.g., the Canadian "shinplasters", 25-cent fractional notes issued in 1870 and in 1900, had no serial number). These considerations make serial numbers a natural leading example of moneyspots. As it will become clear below, the main results of this paper generalize to coarser moneyspots, i.e., to specifications with extraneous attributes that unlike serial numbers, only allow agents to identify proper subsets of notes rather than each individual note.
} 
period $t$ entitles the bearer to collect a note in period $t+1$. The stock of bonds outstanding at the beginning of period $t$ is represented by the set $\mathcal{B}_{t}=\left[0, B_{t}\right]$, where $B_{t} \in \mathbb{R}^{+}$. Every bond outstanding in period $t$ is different from every outstanding note, and uniquely identified by a serial number $s \in \mathcal{B}_{t}{ }^{3}$

The timing of government interventions in a typical period $t$ is as follows. The set of bonds outstanding at time $t, \mathcal{B}_{t}$, is redeemed after the round of decentralized trade, right before agents trade in the centralized market of period $t$. The new bond issue, $\mathcal{B}_{t+1}$, is sold competitively for notes after the round of centralized trade of period $t$, right before agents enter period $t+1$. The government finances bond redemptions with notes acquired in previous bond sales, and if these notes were not enough, it makes up the difference by printing new notes. ${ }^{4}$ Each newly printed note is marked with a unique serial number (notes are numbered consecutively), but newly printed notes are otherwise identical to pre-existing notes. Let $\mathcal{N}_{t+1}^{-}$denote the set of notes withdrawn from private circulation by the bond sale of period $t$, and let $\mathcal{N}_{t+1}^{+}$be the set of notes injected by the corresponding bond redemption in period $t+1$. Let $\mathcal{M}_{t}$ represent the set of notes outstanding at the beginning of period $t$, and let $\overline{\mathcal{M}}_{t}$ be the set of notes outstanding at the end of the centralized trading session of period $t$, before the new bond sale, i.e., $\overline{\mathcal{M}}_{t}=\mathcal{M}_{t} \cup \mathcal{N}_{t}^{+}$. The law of motion for the post-redemption set of outstanding notes is

$$
\overline{\mathcal{M}}_{t+1}=\left(\overline{\mathcal{M}}_{t} \backslash \mathcal{N}_{t+1}^{-}\right) \cup \mathcal{N}_{t+1}^{+}
$$

Let $M_{t}$ represent the size of the set of notes outstanding at the beginning of period $t$, and let $\bar{M}_{t}$ be the size of the set of notes outstanding at the end of the centralized trading session of period $t$, before the new bond sale. Since $B_{t}$ is the size of the set of bonds outstanding at the beginning of period $t, \bar{M}_{t}=M_{t}+B_{t}$. Intuitively, $B_{t}, M_{t}$, and $\bar{M}_{t}$ represent the period- $t$ quantities of bonds, notes outstanding at the beginning of the period, and notes outstanding after that period's bond redemption, respectively. Formally, consider the measure space $\left(\mathbb{R}^{+}, \mathcal{F}\left(\mathbb{R}^{+}\right), \mu\right)$, where $\mathcal{F}\left(\mathbb{R}^{+}\right)$denotes the Borel $\sigma$-field on $\mathbb{R}^{+}$, and $\mu$ is the Lebesgue measure. Then $M_{t}=\mu\left(\mathcal{M}_{t}\right), \bar{M}_{t}=\mu\left(\overline{\mathcal{M}}_{t}\right)$, and $B_{t}=\mu\left(\mathcal{B}_{t}\right)=\mu\left(\mathcal{N}_{t}^{+}\right){ }^{5} \quad \mathrm{~A}$

\footnotetext{
${ }^{3}$ For example, notes are green while bonds are blue. Whether or not every bond is assumed to be indistinguishable from every other bond is inessential for the main results; the main proposition goes through even if every bond is assumed to be identical to every other bond.

${ }^{4}$ The analysis will abstract from fiscal considerations, in particular, I will not assume that the monetary authority has the ability to levy lump-sum taxes.

${ }^{5}$ Throughout, the maintained assumption will be that $\mathcal{M}_{t}, \overline{\mathcal{M}}_{t}, \mathcal{N}_{t}^{+}$, and $\mathcal{N}_{t}^{-}$are elements of $\mathcal{F}\left(\mathbb{R}^{+}\right)$. This does not entail any substantial restriction on individual behavior, and is inconsequential for the economic results.
} 
bond issue $\mathcal{B}_{t+1}$ injects $B_{t+1}$ notes in period $t+1$, and withdraws from private circulation $\mu\left(\mathcal{N}_{t+1}^{-}\right)=\int_{\mathcal{B}_{t+1}} q_{t}(s) \mu(d s) \equiv Q_{t}\left(\mathcal{B}_{t+1}\right)$ notes at the end of period $t$, where $q_{t}(s)$ is interpreted as the quantity of notes needed to purchase a bond with serial number $s$ at time $t .{ }^{6}$ Formally, let $\mathcal{F}\left(\mathcal{B}_{t}\right)=\left\{\mathcal{B}^{\prime} \in \mathcal{F}\left(\mathbb{R}^{+}\right): \mathcal{B}^{\prime} \subseteq \mathcal{B}_{t}\right\}$, then $q_{t}: \mathcal{B}_{t+1} \rightarrow \mathbb{R}^{+} \cup \infty$ is a $\mathcal{F}\left(\mathcal{B}_{t+1}\right)$-measurable function. The law of motion for the measure of the post-redemption set of outstanding notes is

$$
\bar{M}_{t+1}=\bar{M}_{t}-Q_{t}\left(\mathcal{B}_{t+1}\right)+B_{t+1} .
$$

The size of the bond issue, $B_{t+1}$, expressed relative to the size of the post-redemption money supply, $\bar{M}_{t}$, will be denoted by $x_{t}$. That is, if there are $\bar{M}_{t}$ notes outstanding after the round of centralized trade, at the end of the period the government auctions off claims to $B_{t+1}=x_{t} \bar{M}_{t}$ notes payable at the beginning of the centralized trading session one period hence. Note that if $q_{t}(s)=q_{t}$ for all $s$, then $\bar{M}_{t+1}=\bar{M}_{t}-q_{t} B_{t+1}+B_{t+1}$, and

$$
\bar{M}_{t+1}=\left[1+\left(1-q_{t}\right) x_{t}\right] \bar{M}_{t}
$$

The motion of $x_{t}$ is assumed to follow a Markov process with transition function $F\left(x^{\prime}, x\right)=$ $\operatorname{Pr}\left(x_{t+1} \leq x^{\prime} \mid x_{t}=x\right)$, where $F: \mathbb{R}^{+} \times \mathbb{R}^{+} \rightarrow \mathbb{R}$ is continuous. For each fixed $x, F(\cdot, x)$ is a distribution function with support $\mathbb{X} \subset \mathbb{R}^{+}$. It is assumed that the process defined by $F$ has a stationary distribution $\psi$, the unique solution to $\psi\left(x^{\prime}\right)=\int F\left(x^{\prime}, x\right) d \psi(x)$, and that $F$ has the Feller property, i.e., for any continuous real-valued function $g$ on $\mathbb{X}, \int g\left(x^{\prime}\right) d F\left(x^{\prime}, x\right)$ is a continuous function of $x$. The realization $x_{t}$ is observed by all agents and is common knowledge at the beginning of period $t$ (before the round of decentralized trade). Aside from these stochastic open-market operations, there are no shocks to the fundamentals of the economy.

Let the utility function for special goods, $u: \mathbb{R}^{+} \rightarrow \mathbb{R}$, be continuously differentiable, increasing, and strictly concave, with $u(0)=0$. Suppose there exists $c^{*} \in(0, \infty)$ defined by $u^{\prime}\left(c^{*}\right)=1$, with $c^{*} \leq \bar{h}$. Each agent ranks consumption and labor supply bundles according to

$$
E_{0}\left\{\sum_{t=0}^{\infty} \beta^{t}\left[u\left(c_{t}\right)-l_{t}+y_{t}-h_{t}\right]\right\},
$$

\footnotetext{
${ }^{6}$ I will work under the assumption that the government treats notes symmetrically regardless of serial number. One could imagine that the government gives certain serial numbers differential treatment in open-market operations, e.g., by requiring that bond purchases be paid with those specific serial numbers. I will not explore such formulations, but as it will become clear below, the main insights would not be affected. In what follows, given a $\mathcal{F}\left(\mathbb{R}^{+}\right)$-measurable function $f$, I will denote the Lebesgue integral $\int f(n) \mu(d n)$ by $\int f(n) d \mu$, or when there is no possbility of confusion, by the more concise $\int f d \mu$.
} 
where $\beta \in(0,1), c_{t}$ and $l_{t}$ are the quantities of special goods consumed and produced in the decentralized market, $y_{t}$ denotes consumption of general goods, and $h_{t}$ represents the hours worked in the second subperiod. ${ }^{7} E_{t}$ is the expectations operator conditional on the information available to the agent at time $t$, defined with respect to the matching probabilities and the probability measure over $\left\{x_{t}\right\}$ induced by $F$.

\section{The impossibility proposition}

In this section I focus on the special case in which notes have no extraneous attributes, i.e., there are no serial numbers, so every note is indistinguishable from every other note. In this context, I establish a well-known impossibility result, namely, that there cannot exist a monetary equilibrium in which money coexists with nominal default-free, interest-bearing bonds. ${ }^{8}$

Begin by describing the individual optimization problems faced by an agent in a typical period, starting at the end of the period. Consider an agent who enters the competitive market for government bonds at the end of period $t$ with $\bar{m}_{t} \in \mathbb{R}^{+}$notes. His problem consists of choosing a portfolio $\boldsymbol{a}_{t+1}=\left(m_{t+1}, b_{t+1}\right)$, where $b_{t+1}$ is the quantity of bonds he purchases, and $m_{t+1}$ is the quantity of notes he holds after the bond purchase. Formally, in the market for government bonds this agent solves

$$
\begin{gathered}
U_{t}\left(\bar{m}_{t}\right)=\max _{\boldsymbol{a}_{t+1}} \beta E_{t} V_{t+1}\left(\boldsymbol{a}_{t+1}\right) \\
\text { s.t. } q_{t} b_{t+1}+m_{t+1} \leq \bar{m}_{t}, m_{t+1}, b_{t+1} \in \mathbb{R}^{+},
\end{gathered}
$$

where $q_{t}$ is the money price of a bond, and $V_{t+1}\left(\boldsymbol{a}_{t+1}\right)$ is the maximum expected utility that the agent can attain by entering the decentralized market of period $t+1$ with portfolio $\boldsymbol{a}_{t+1}$. Let $W_{t}\left(\boldsymbol{a}_{t}\right)$ denote the maximum expected discounted utility that an agent can attain when he enters the centralized market holding a portfolio $\boldsymbol{a}_{t}$ in period $t$. This value satisfies

$$
\begin{gathered}
W_{t}\left(\boldsymbol{a}_{t}\right)=\max _{y_{t}, h_{t}, \bar{m}_{t}}\left\{y_{t}-h_{t}+U_{t}\left(\bar{m}_{t}\right)\right\} \\
\text { s.t. } y_{t}-h_{t}+\phi_{t} \bar{m}_{t}=\phi_{t}\left(m_{t}+b_{t}\right), y_{t}, h_{t}, \bar{m}_{t} \in \mathbb{R}^{+},
\end{gathered}
$$

\footnotetext{
${ }^{7}$ Many of the assumptions on preferences are made for convenience and can be relaxed without changing anything of substance. All that is needed for analytical tractability is that the period utility function is quasilinear in $y_{t}$ or $h_{t}$ (see Lagos and Wright, 2005).

${ }^{8}$ Versions of this impossibility result in the context of other models can be found, for instance, in Aiyagari, Wallace and Wright (1996), Hellwig (1993), Kocherlakota (2003), and Wallace (1983, 1990).
} 
and $h_{t} \leq \bar{h}$, where $\phi_{t}$ is the price of a note in terms of general goods. On the left side of the budget constraint are the agent's net consumption of general goods and the real value of the money holdings he is choosing. On the right side is the real value of his post-redemption money holdings. ${ }^{9}$

Consider a bilateral meeting in the decentralized market of period $t$ between a buyer and a seller who hold portfolios $\left(m_{t}, b_{t}\right)$, and $\left(\hat{m}_{t}, \hat{b}_{t}\right)$, respectively. A bargaining outcome is a quantity of special good, $c_{t}$, that the seller produces for the buyer in exchange for a portfolio of notes and bonds, $\left(m_{t}^{\prime}, b_{t}^{\prime}\right)$, that the buyer offers as payment. The gains from trade from such an outcome would be $u\left(c_{t}\right)+W_{t}\left(m_{t}-m_{t}^{\prime}, b_{t}-b_{t}^{\prime}\right)-W_{t}\left(m_{t}, b_{t}\right)$ for the buyer, and $-c_{t}+W_{t}\left(\hat{m}_{t}+m_{t}^{\prime}, \hat{b}_{t}+\right.$ $\left.b_{t}^{\prime}\right)-W_{t}\left(\hat{m}_{t}, \hat{b}_{t}\right)$ for the seller. Hence, the buyer's take-it-or-leave-it offer solves

$$
\max _{c_{t}, m_{t}^{\prime}, b_{t}^{\prime}}\left\{u\left(c_{t}\right)-\phi_{t}\left(m_{t}^{\prime}+b_{t}^{\prime}\right)\right\} \quad \text { s.t. } c_{t} \leq \phi_{t}\left(m_{t}^{\prime}+b_{t}^{\prime}\right), 0 \leq c_{t} \leq \bar{h}, m_{t}^{\prime} \leq m_{t}, b_{t}^{\prime} \leq b_{t} .
$$

The first constraint requires that the trade be individually rational for the seller, and the last two constraints state that the buyer can only pay with the notes and bonds that he owns. The bargaining outcome can be described as follows. If $c^{*} \leq \phi_{t}\left(m_{t}+b_{t}\right)$, the buyer exchanges some portion $\left(m_{t}^{\prime}, b_{t}^{\prime}\right)$ of his portfolio with value $\phi_{t}\left(m_{t}^{\prime}+b_{t}^{\prime}\right)=c^{*}$ for a quantity $c^{*}$ of the special good. Else, the buyer gives the seller his whole portfolio, i.e., $\left(m_{t}^{\prime}, b_{t}^{\prime}\right)=\left(m_{t}, b_{t}\right)$, in exchange for $c_{t}=\phi_{t}\left(m_{t}+b_{t}\right)$ special goods. Hence, the quantity traded can be written as a function $c: \mathbb{R}^{+} \rightarrow\left[0, c^{*}\right]$ of the real value of the buyer's portfolio, $\phi_{t}\left(m_{t}+b_{t}\right)$. Specifically, the quantity traded in the decentralized market of period $t$ between a buyer who holds portfolio $\left(m_{t}, b_{t}\right)$ and a seller, is $c_{t}=c\left(\phi_{t}\left(m_{t}+b_{t}\right)\right)$, where

$$
c(x) \equiv \min \left(x, c^{*}\right)
$$

The maximum expected discounted utility attainable by an agent who enters the decentralized market of period $t$ with portfolio $\boldsymbol{a}_{t}$ can be written as

$$
V_{t}\left(\boldsymbol{a}_{t}\right)=\mathcal{L}\left[\phi_{t}\left(m_{t}+b_{t}\right)\right]+W_{t}(0)
$$

where

$$
\mathcal{L}(x) \equiv \alpha\{u[c(x)]+x-c(x)\}+(1-\alpha) x
$$

\footnotetext{
${ }^{9}$ To simplify the exposition, it is convenient to abstract from the agent's decision of whether to redeem a bond for a note, and simply assume that all bonds are redeemed at maturity. A mechanical way to think about this would be to imagine that the bond automatically turns into a note at maturity. Below, I will discuss the implications of endogenizing the agents' decision of whether and when to redeem their bond holdings.
} 
is the expected utility that an agent obtains over both subperiods of any given period, if he enters the first subperiod with a portfolio of money and nominal bonds of real value $x$ (expressed in terms of current-period general goods). ${ }^{10}$ To interpret $\mathcal{L}(x)$, note that with probability $\alpha$, the agent acts as a buyer in the first subperiod: the portfolio allows him to buy $c(x)$ special goods (which give him utility $u[c(x)]$ ), and the real value of the unspent portfolio, $x-c(x)$, can be sold for $x-c(x)$ general goods in the following centralized market. With probability $1-\alpha$, the agent has no opportunity to use his portfolio in a bilateral exchange, and he carries it into the centralized market where he can sell it for $x$ general goods. Equivalently, since $x$ is the real resale value of the portfolio that the agent carries into the period, and given that the expected gain from trade in the decentralized market, $\alpha\{u[c(x)]-c(x)\}$, can be interpreted as an expected "liquidity dividend" from holding the portfolio, one can write $\mathcal{L}(x)=x+\alpha\{u[c(x)]-c(x)\}$, and interpret $\mathcal{L}(x)$ as the cum (liquidity) dividend expected value (in terms of current-period general goods) of the portfolio of money and bonds. ${ }^{11}$

Definition 1 An equilibrium of the economy with no extraneous attributes is an allocation $\left\{\left(y_{i t}, h_{i t}, \bar{m}_{i t}, m_{i t+1}, b_{i t+1}\right)_{i \in \mathcal{I}}\right\}_{t=0}^{\infty}$, bargaining outcomes $\left\{\left(c_{i j t}\right)_{i, j \in \mathcal{I}}\right\}_{t=0}^{\infty}$, and prices $\left\{\phi_{t}, q_{t}\right\}_{t=0}^{\infty}$, such that: (i) given prices and the bargaining protocol, for all $i \in \mathcal{I},\left\{y_{i t}, h_{i t}, \bar{m}_{i t}, m_{i t+1}, b_{i t+1}\right\}_{t=0}^{\infty}$ solves agent $i$ 's optimization problem in the competitive markets; (ii) the bilateral terms of trade are determined by Nash bargaining, i.e., if agent $i$ is the buyer and agent $j$ the seller in a bilateral meeting at time $t$, then $j$ produces $c_{i j t}=\min \left[\phi_{t}\left(m_{i t}+b_{i t}\right), c^{*}\right]$ special goods for $i\left(c_{i j t}=0\right.$ if $i$ and $j$ are not in a single-coincidence meeting at $t$ ); and (iii) the centralized markets clear for all $t$. An equilibrium is said to be "monetary" if $\phi_{t}>0$ for all $t$, and in this case the allocation must also satisfy: (a) the money-market clearing condition, $\int_{\mathcal{I}} \bar{m}_{i t} d \mu=\bar{M}_{t}$, and (b) the bondmarket clearing condition, $\int_{\mathcal{I}} b_{i t+1} d \mu=B_{t+1}$, for all $t \geq 0$. In a monetary equilibrium, money is said to coexist with bonds in period $t$, if $B_{t}>0$ and $\int_{\mathcal{I}} \phi_{t} m_{i t} d \mu>0$.

Proposition 1 Let $i_{t+1}=1 / q_{t}-1$ denote the nominal interest rate on nominal bonds between period $t$ and period $t+1$. The set of monetary equilibria of the economy with no extraneous attributes is nonempty if $u^{\prime}(0)>1+\frac{1-\beta}{\alpha \beta}$. In any monetary equilibrium of the economy with no extraneous attributes, money coexists with bonds in period $t$ only if $i_{t+1}=0$.

\footnotetext{
${ }^{10}$ The symbol 0 will be used to denote the origin in $\mathbb{R}^{k}$ as well as the real number zero; no confusion will result.

${ }^{11}$ Notice that $\mathcal{L}(x)$ is twice differentiable everywhere, with $\mathcal{L}^{\prime}(x)=1+\alpha\left[u^{\prime}(x)-1\right] \mathbb{I}_{\left\{x<c^{*}\right\}}$, and $\mathcal{L}^{\prime \prime}(x)=$ $\alpha u^{\prime \prime}(x) \mathbb{I}_{\left\{x<c^{*}\right\}}$, where $\mathbb{I}_{\left\{x<c^{*}\right\}}$ is an indicator function that takes the value 1 if $x<c^{*}$.
} 
Each bond issued at the end of period $t$ becomes a note with certainty after the round of decentralized trade of period $t+1$. Agents anticipate this fact in the decentralized market of period $t+1$, and therefore value a bond and a note identically at that point. When agents choose their money and bonds portfolio in the bond market of period $t$, they anticipate that a note and a bond will be treated as the same asset by everyone in the following period, so they choose to hold no money if $q_{t}<1$, and are indifferent between holding money and bonds if $q_{t}=1$. (Bond-market clearing is inconsistent with $q_{t}>1$.) A monetary equilibrium may exhibit periods with $q_{t}=1$ and periods with $q_{t}<1$. Money will circulate (alongside bonds), or equivalently, money and bonds will coexist, e.g., in the portfolios that agents choose to carry into the decentralized market, only in periods when the nominal interest rate is zero. In any period $t$ with $1 / q_{t}-1>0$, agents use all their notes to purchase government bonds, and enter period $t+1$ holding no money. Hence, in such a period $t+1$, money does not circulate: only bonds are used as medium of exchange in decentralized trades. Proposition 1 is a version of a well-known result: Money cannot coexist with interest-bearing default-free nominal bonds in the portfolio of assets held by the private sector. ${ }^{12}$

\section{Coexistence of money and interest-bearing nominal bonds}

In this section I consider the general setup with nominal bonds and notes with extraneous attributes. In this context, I establish the existence of monetary equilibria where money coexists with interest-bearing nominal bonds.

The set of post-redemption notes outstanding during the centralized trading session of period $t$ is $\overline{\mathcal{M}}_{t} \subset \mathbb{R}^{+}$. Let $\mathcal{F}\left(\overline{\mathcal{M}}_{t}\right)=\left\{\mathcal{M} \in \mathcal{F}\left(\mathbb{R}^{+}\right): \mathcal{M} \subseteq \overline{\mathcal{M}}_{t}\right\}$, and use finite, nonnegative measures on $\mathcal{F}\left(\overline{\mathcal{M}}_{t}\right)$ to represent portfolios of time- $t$ post-redemption notes. Specifically, let $\overline{\mathfrak{m}}_{t}$ denote a finite nonnegative measure on $\mathcal{F}\left(\overline{\mathcal{M}}_{t}\right)$, and let $\overline{\mathfrak{M}}_{t}$ denote the collection of all such measures, then $\overline{\mathfrak{m}}_{i t} \in \overline{\mathfrak{M}}_{t}$ is the portfolio of post-redemption notes held by agent $i$ at time $t$. Similarly, let $\mathfrak{M}_{t}$ denote the collection of all finite nonnegative measures on $\mathcal{F}\left(\mathcal{M}_{t}\right)=$

\footnotetext{
${ }^{12}$ This is so, of course, as long as the environment does not give money an advantage relative to bonds in the exchange process. For example, if one exogenously imposes that agents can only use money as a medium of exchange, then it is easy to construct a monetary equilibrium in which money circulates in periods when bonds pay interest. Such exogenous restrictions on the use of bonds as media of exchange seem to be the standard assumption underlying cash-in-advance models, as well as an assumption used in some versions of monetary search models with multiple assets. As another example, Zhu and Wallace (2007) obtain coexistence of money and interest-bearing nominal bonds by assuming a bilateral trading protocol that effectively confers larger gains from trade to buyers who hold a larger proportion of money in their portfolios.
} 
$\left\{\mathcal{M} \in \mathcal{F}\left(\mathbb{R}^{+}\right): \mathcal{M} \subseteq \mathcal{M}_{t}\right\}$, and let $\mathfrak{m}_{i t}$ be a typical element, interpreted as the beginning-ofperiod- $t$ portfolio of notes held by agent $i$. Also, let $\mathfrak{B}_{t}$ be the set of all finite nonnegative measures on $\mathcal{F}\left(\mathcal{B}_{t}\right)$, and use $\mathfrak{b}_{i t+1} \in \mathfrak{B}_{t+1}$ to represent the portfolio of bonds held by agent $i$ at the end of period $t$. In general, given a set of assets $\mathcal{S}$ with associated Borel $\sigma$-algebra $\mathcal{F}(\mathcal{S})$, a real-valued $\mathcal{F}(\mathcal{S})$-measurable price function $p$ on $\mathcal{S}$, and a portfolio $\mathfrak{f}$ which is an element of the collection of all finite nonnegative measures on $\mathcal{F}(\mathcal{S})$, the Walrasian value of the portfolio $\mathfrak{f}$ is computed in the usual way, i.e., $p \cdot \mathfrak{f}=\int_{\mathcal{S}} p(n) \mathfrak{f}(d n) .{ }^{13}$

I proceed by describing the individual optimization problems faced by an agent in a typical period, starting at the end of the period. Consider an agent $i \in \mathcal{I}$ who is holding a portfolio of notes $\overline{\mathfrak{m}}_{i t} \in \overline{\mathfrak{M}}_{t}$ when he enters the competitive market for government bonds at the end of period $t$. This agent's problem consists of choosing measures $\mathfrak{n}_{i t+1}^{-}$and $\mathfrak{a}_{i t+1}=\left(\mathfrak{m}_{i t+1}, \mathfrak{b}_{i t+1}\right)$, where $\mathfrak{b}_{i t+1}$ is the portfolio of bonds that he purchases, $\mathfrak{n}_{i t+1}^{-}$is the portfolio of notes that he trades in the bond market $\left(\mathfrak{n}_{i t+1}^{-}\left(\overline{\mathcal{M}}_{t}\right)\right.$ is the quantity of notes that he uses to pay for the bond purchase), and $\mathfrak{m}_{i t+1}$ is the portfolio of notes that he holds after the bond purchase. The value of this agent's problem in the bond market is

$$
\begin{aligned}
U_{t}\left(\overline{\mathfrak{m}}_{i t}\right)=\max _{\mathfrak{a}_{i t+1}, \mathfrak{n}_{i t+1}^{-}} \beta E_{t} V_{t+1}\left(\mathfrak{a}_{i t+1}\right) & \\
\text { s.t. } \quad \mathfrak{n}_{i t+1}^{-}(\mathcal{M})+\mathfrak{m}_{i t+1}(\mathcal{M}) & \leq \overline{\mathfrak{m}}_{i t}(\mathcal{M}) \quad \text { for all } \mathcal{M} \in \mathcal{F}\left(\overline{\mathcal{M}}_{t}\right) \\
q_{t} \cdot \mathfrak{b}_{i t+1} & \leq \mathfrak{n}_{i t+1}^{-}\left(\overline{\mathcal{M}}_{t}\right) \\
\mathfrak{m}_{i t+1}, \mathfrak{n}_{i t+1}^{-} & \in \overline{\mathfrak{M}}_{t}, \mathfrak{b}_{i t+1} \in \mathfrak{B}_{t+1}
\end{aligned}
$$

For any $\mathfrak{a}_{i t+1}$ with $\mathfrak{m}_{i t+1} \in \overline{\mathfrak{M}}_{t}$ and $\mathfrak{b}_{t+1} \in \mathfrak{B}_{t+1}, V_{t+1}\left(\mathfrak{a}_{t+1}\right)$ denotes the maximum expected discounted utility that the agent can attain when he enters the decentralized market of period $t+1$ holding portfolio $\mathfrak{a}_{t+1}$ (after the size of the open-market operation of period $t+1$ has been announced, but before the realization of the bilateral matching). The first constraint states that the notes that the agent uses to purchase bonds, and the notes that he carries into the next round of decentralized trade, must belong to the portfolio of notes that the agent was holding before the open-market operation. The second constraint states that given the price

\footnotetext{
${ }^{13}$ This formulation is often used in the theory of general Walrasian equilibrium with infinitely many commodities (e.g., Jones, 1983), and it encompasses the following special cases. If $\mathfrak{f}$ has a Radon-Nikodym derivative $f$ with respect to $\mu$, then $p \cdot \mathfrak{f}=\int_{\mathcal{S}} p(n) f(n) \mu(d n)$, and $\mathfrak{f}\left(\mathcal{S}^{\prime}\right)=\int_{\mathcal{S}^{\prime}} f(n) \mu(d n)$ for all $\mathcal{S}^{\prime} \in \mathcal{F}(\mathcal{S})$. If $\mathfrak{f}$ consists of point masses of size $f_{i}$ at $k$ points $n_{1}, \ldots, n_{k} \in \mathcal{S}$ then $p \cdot \mathfrak{f}=\sum_{i=1}^{k} p\left(n_{i}\right) f_{i}$, and $\mathfrak{f}\left(\mathcal{S}^{\prime}\right)=\sum_{i=1}^{k} \mathbb{I}_{\left\{n_{i} \in \mathcal{S}^{\prime}\right\}} f_{i}$. For any two vectors $\boldsymbol{\phi}=\left(\phi^{1}, \ldots, \phi^{k}\right)$ and $\boldsymbol{m}=\left(m^{1}, \ldots, m^{k}\right)$, the expression $\boldsymbol{\phi} \boldsymbol{m}$ denotes the scalar product $\sum_{s=1}^{k} \phi^{s} m^{s}$.
} 
function for bonds, $q_{t}$, the agent has enough notes to finance $\mathfrak{a}_{t+1}$. (Implicit in this constraint is the maintained assumption that the government ignores extraneous attributes.)

As stated in Section 2, each bond represents a sure claim to a note. However, since notes are differentiated by serial numbers, in order to price a bond, it may not be enough for an agent to know that the bond will be redeemed with a note: the agent may also wish to predict the serial number of the note that will be used to redeem a particular bond. For this reason, a government redemption lottery is specified for each period $t$. Intuitively, a redemption lottery is a probability measure over assignments of the notes that the government uses for redemption to bonds, specifying the probability that a collection of bonds with serial numbers in a (Borel measurable) set $\mathcal{B}^{\prime} \subseteq \mathcal{B}_{t}$ is redeemed with a set of notes with serial numbers in the (Borel measurable) set $\mathcal{N}^{\prime} \subseteq \mathcal{N}_{t}^{+}$. Let $\Omega_{t}=\left\{\omega: \mathcal{B}_{t} \rightarrow \mathcal{N}_{t}^{+} \mid \omega(\varnothing)=\varnothing, \omega\right.$ is bijective, $\mu$-measure preserving, and Borel measurable\}. A time-t assignment (of notes to bonds) is an element $\omega \in \Omega_{t}$, i.e., an assignment maps measurable sets of outstanding bonds onto measurable sets of notes (of equal size) being used for redemption. That is, each function $\omega \in \Omega_{t}$, assigns a set $\mathcal{N}^{\prime} \subseteq \mathcal{N}_{t}^{+}$to each $\mathcal{B}^{\prime} \subseteq \mathcal{B}_{t}$ via $\mathcal{N}^{\prime}=\left\{n \in \mathcal{N}_{t}^{+} \mid n=\omega(s)\right.$ for $\left.s \in \mathcal{B}^{\prime}\right\}$, or more compactly, via $\mathcal{N}^{\prime}=\omega\left(\mathcal{B}^{\prime}\right)$. Let $\mathcal{F}_{\Omega_{t}}$ denote the $\sigma$-field generated by $\Omega_{t}$, and let $\nu_{t}$ be a probability measure on $\mathcal{F}_{\Omega_{t}}$. The probability space $\left(\Omega_{t}, \mathcal{F}_{\Omega_{t}}, \nu_{t}\right)$ represents the government redemption lottery at time $t$. The period- $t$ assignment (i.e., the realization $\omega \in \Omega_{t}$ ) becomes known after the round of decentralized trade, right before the bonds are redeemed.

Let $\phi_{t}: \overline{\mathcal{M}}_{t} \rightarrow \mathbb{R}^{+} \cup \infty$ be a $\mathcal{F}\left(\overline{\mathcal{M}}_{t}\right)$-measurable function. Intuitively, $\phi_{t}$ is the price function for notes at time $t$, i.e., $\phi_{t}(n)$ is interpreted as the price in terms of general goods of a note with serial number $n$. For a given $\mathfrak{a}_{i t}=\left(\mathfrak{m}_{i t}, \mathfrak{b}_{i t}\right)$ with $\mathfrak{m}_{i t} \in \overline{\mathfrak{M}}_{t-1}$ and $\mathfrak{b}_{i t} \in \mathfrak{B}_{t}$, let $W_{t}\left(\mathfrak{a}_{i t}\right)$ denote the maximum expected discounted utility that an agent $i \in \mathcal{I}$ can attain when he enters the time- $t$ centralized market (after the realization of the redemption lottery has been observed) with $\mathfrak{a}_{i t}$. This value satisfies

$$
\begin{gathered}
W_{t}\left(\mathfrak{a}_{i t}\right)=\max _{y_{i t}, h_{i t}, \overline{\mathfrak{m}}_{i t}}\left\{y_{i t}-h_{i t}+U_{t}\left(\overline{\mathfrak{m}}_{i t}\right)\right\} \\
\text { s.t. } y_{i t}-h_{i t}+\phi_{t} \cdot \overline{\mathfrak{m}}_{i t}=\phi_{t} \cdot \mathfrak{m}_{i t}+\phi_{t} \cdot \mathfrak{n}_{i t}^{+},
\end{gathered}
$$

$y_{i t}, h_{i t} \in \mathbb{R}^{+}, \overline{\mathfrak{m}}_{i t} \in \overline{\mathfrak{M}}_{t}$, and $h_{i t} \leq \bar{h}$, where $\phi_{t} \cdot \mathfrak{m}_{i t}=\int_{\mathcal{M}_{t}} \phi_{t}(n) \mathfrak{m}_{i t}(d n)$, and $\phi_{t} \cdot \mathfrak{n}_{i t}^{+} \equiv$ $\int_{\mathcal{N}_{t}^{+}} \phi_{t}(n) \mathfrak{n}_{i t}^{+}(d n)$. The measure $\mathfrak{n}_{i t}^{+}$represents the portfolio of notes that the agent receives from the government upon redeeming his bond holdings, $\mathfrak{b}_{i t}$. Formally, let $\mathcal{F}\left(\mathcal{N}_{t}^{+}\right)$denote the Borel $\sigma$-field on $\mathcal{N}_{t}^{+}$, then $\mathfrak{n}_{i t}^{+}$is the finite nonnegative measure defined by $\mathfrak{n}_{i t}^{+}(\mathcal{N})=\mathfrak{b}_{i t}\left(\omega^{-1}(\mathcal{N})\right)$ 
for each $\mathcal{N} \in \mathcal{F}\left(\mathcal{N}_{t}^{+}\right) .{ }^{14}$ Hence, the second integral on the right side of the budget constraint can be written as $\phi_{t} \cdot \mathfrak{n}_{i t}^{+}=\int_{\mathcal{N}_{t}^{+}} \phi_{t}(n) \mathfrak{b}_{i t}\left(\omega^{-1}(d n)\right) \equiv \phi_{t} \cdot\left(\mathfrak{b}_{i t} \omega^{-1}\right)$, and represents the real value (in terms of general goods) of the portfolio of notes that the agent receives from the government upon redeeming his bond holdings, $\mathfrak{b}_{i t}$. The first integral on the right side of the budget constraint represents the real value (in terms of general goods) of the portfolio of notes that the agent brought into the decentralized market. On the left side of the budget constraint, the difference $y_{i t}-h_{i t}$ represents the net quantity of general goods consumed (or produced, if negative), and the integral is the real value of the set of notes that the agent is choosing.

Consider a bilateral meeting in the decentralized market of period $t$ between a buyer $i \in$ $\mathcal{I}$ and a seller $j \in \mathcal{I}$ who are holding portfolios $\left(\mathfrak{m}_{i t}, \mathfrak{b}_{i t}\right)$ and $\left(\mathfrak{m}_{j t}, \mathfrak{b}_{j t}\right)$, respectively, where $\mathfrak{m}_{i t}, \mathfrak{m}_{j t} \in \mathfrak{M}_{t}$, and $\mathfrak{b}_{i t}, \mathfrak{b}_{j t} \in \mathfrak{B}_{t}$. A bargaining outcome is a quantity of special good, $c_{i j t}$, that the seller produces for the buyer in exchange for a portfolio $\left(\mathfrak{m}_{t}^{\prime}, \mathfrak{b}_{t}^{\prime}\right)$, with $\mathfrak{m}_{t}^{\prime} \in \mathfrak{M}_{t}$, and $\mathfrak{b}_{t}^{\prime} \in \mathfrak{B}_{t}$, that the buyer offers as payment. Define the measures $\mathfrak{m}_{j t} \oplus \mathfrak{m}^{\prime}, \mathfrak{m}_{i t} \ominus \mathfrak{m}^{\prime}, \mathfrak{b}_{j t} \oplus \mathfrak{b}^{\prime}$, and $\mathfrak{b}_{i t} \ominus \mathfrak{b}^{\prime}$, by $\mathfrak{m}_{j t} \oplus \mathfrak{m}^{\prime}(\mathcal{M})=\mathfrak{m}_{j t}(\mathcal{M})+\mathfrak{m}^{\prime}(\mathcal{M})$, and $\mathfrak{m}_{i t} \ominus \mathfrak{m}^{\prime}(\mathcal{M})=\mathfrak{m}_{i t}(\mathcal{M})-\mathfrak{m}^{\prime}(\mathcal{M})$ for each $\mathcal{M} \in \mathcal{F}\left(\mathcal{M}_{t}\right)$, and by $\mathfrak{b}_{j t} \oplus \mathfrak{b}^{\prime}(\mathcal{B})=\mathfrak{b}_{j t}(\mathcal{B})+\mathfrak{b}^{\prime}(\mathcal{B})$, and $\mathfrak{b}_{i t} \ominus \mathfrak{b}^{\prime}(\mathcal{B})=\mathfrak{b}_{i t}(\mathcal{B})-\mathfrak{b}^{\prime}(\mathcal{B})$ for each $\mathcal{B} \in \mathcal{F}\left(\mathfrak{B}_{t}\right)$. Note that $\mathfrak{m}_{j t} \oplus \mathfrak{m}^{\prime} \in \mathfrak{M}_{t}$ and $\mathfrak{b}_{j t} \oplus \mathfrak{b}^{\prime} \in \mathfrak{B}_{t}$, while $\mathfrak{m}_{i t} \ominus \mathfrak{m}^{\prime}$ and $\mathfrak{b}_{i t} \ominus \mathfrak{b}^{\prime}$ are finite signed measures on $\mathcal{F}\left(\mathcal{M}_{t}\right)$, and $\mathcal{F}\left(\mathcal{B}_{t}\right)$, respectively. The gains from trade from a bargaining outcome $\left(c_{i j t}, \mathfrak{m}_{t}^{\prime}, \mathfrak{b}_{t}^{\prime}\right)$ would be $u\left(c_{i j t}\right)+E_{t}\left[W_{t}\left(\mathfrak{m}_{i t} \ominus \mathfrak{m}_{t}^{\prime}, \mathfrak{b}_{i t} \ominus \mathfrak{b}_{t}^{\prime}\right)\right]-E_{t}\left[W_{t}\left(\mathfrak{m}_{i t}, \mathfrak{b}_{i t}\right)\right]$ for the buyer, and $-c_{i j t}+E_{t}\left[W_{t}\left(\mathfrak{m}_{j t} \oplus \mathfrak{m}_{t}^{\prime}, \mathfrak{b}_{j t} \oplus \mathfrak{b}_{t}^{\prime}\right)\right]-E_{t}\left[W_{t}\left(\mathfrak{m}_{j t}, \mathfrak{b}_{j t}\right)\right]$ for the seller, where the expectation is with respect to the measure $\nu_{t}$, over assignment realizations of the redemption lottery. The buyer's take-it-or-leave-it offer solves

$$
\max _{c_{t}, \mathfrak{m}_{t}^{\prime}, \mathfrak{b}_{t}^{\prime}} u\left(c_{t}\right)-\Lambda_{t}\left(\mathfrak{m}_{t}^{\prime}, \mathfrak{b}_{t}^{\prime}\right) \quad \text { s.t. } c_{t} \leq \Lambda_{t}\left(\mathfrak{m}_{t}^{\prime}, \mathfrak{b}_{t}^{\prime}\right), 0 \leq c_{t} \leq \bar{h}, \mathfrak{m}_{i t} \ominus \mathfrak{m}_{t}^{\prime} \in \mathfrak{M}_{t}, \mathfrak{b}_{i t} \ominus \mathfrak{b}_{t}^{\prime} \in \mathfrak{B}_{t}
$$

where $\Lambda_{t}\left(\mathfrak{m}_{t}^{\prime}, \mathfrak{b}_{t}^{\prime}\right) \equiv \phi_{t} \cdot \mathfrak{m}_{t}^{\prime}+\int_{\Omega_{t}} \phi_{t} \cdot\left(\mathfrak{b}_{t}^{\prime} \omega^{-1}\right) \nu_{t}(d \omega) .{ }^{15} \quad$ The first constraint requires that the trade be individually rational for the seller. The last two constraints indicate that the buyer can only pay with the notes and bonds that he owns. The bargaining outcome is as follows. If $c^{*} \leq \Lambda_{t}\left(\mathfrak{m}_{i t}, \mathfrak{b}_{i t}\right)$, the buyer exchanges a part $\left(\mathfrak{m}_{t}^{\prime}, \mathfrak{b}_{t}^{\prime}\right)$ of his asset portfolio with value $\Lambda_{t}\left(\mathfrak{m}_{t}^{\prime}, \mathfrak{b}_{t}^{\prime}\right)=$ $c^{*}$, for a quantity $c^{*}$ of the special good. Else, he gives the seller all his asset holdings, i.e., $\left(\mathfrak{m}_{t}^{\prime}, \mathfrak{b}_{t}^{\prime}\right)=\left(\mathfrak{m}_{i t}, \mathfrak{b}_{i t}\right)$, in exchange for $c_{i j t}=\Lambda_{t}\left(\mathfrak{m}_{i t}, \mathfrak{b}_{i t}\right)$ special goods. Hence, the quantity traded in a single-coincidence meeting is $c\left(\Lambda_{t}\left(\mathfrak{m}_{i t}, \mathfrak{b}_{i t}\right)\right)$. With this bargaining solution, (10), and (11),

\footnotetext{
${ }^{14}$ Note that $\omega^{-1}(\mathcal{N}) \in \mathcal{F}\left(\mathcal{B}_{t}\right)$ for any $\mathcal{N} \in \mathcal{F}\left(\mathcal{N}_{t}^{+}\right)$because $\omega$ is Borel measurable.

${ }^{15}$ In the proof of Proposition 2, I will show that $\phi_{t} \cdot\left(\mathfrak{b}_{t}^{\prime} \omega^{-1}\right)$ is $\mathcal{F}_{\Omega_{t}}$-measurable, and that $\int_{\Omega_{t}} \phi_{t} \cdot\left(\mathfrak{b}_{t}^{\prime} \omega^{-1}\right) \nu_{t}(d \omega)$ is well-defined in the class of equilibria of interest.
} 
the maximum expected discounted utility attainable by an agent $i$ who enters the decentralized market of period $t$ holding $\mathfrak{a}_{i t}=\left(\mathfrak{m}_{i t}, \mathfrak{b}_{i t}\right)$, with $\mathfrak{m}_{i t} \in \overline{\mathfrak{M}}_{t-1}$ and $\mathfrak{b}_{i t} \in \mathfrak{B}_{t}$, can be written as

$$
V_{t}\left(\mathfrak{a}_{i t}\right)=\mathcal{L}\left[\Lambda_{t}\left(\mathfrak{a}_{i t}\right)\right]+K_{t}
$$

where $\left\{K_{t}\right\}_{t=0}^{\infty}$ satisfies $K_{t}=\Delta_{t}+\beta E_{t} K_{t+1}$, and

$$
\Delta_{t} \equiv \max _{\overline{\mathfrak{m}}_{i t} \in \overline{\mathfrak{M}}_{t}}\left\{-\phi_{t} \cdot \overline{\mathfrak{m}}_{i t}+\max _{\mathfrak{a}_{i t+1}, \mathfrak{n}_{i t+1}^{-}} \beta E_{t} \mathcal{L}\left[\Lambda_{t+1}\left(\mathfrak{a}_{i t+1}\right)\right]\right\}
$$

subject to (7), (8), and (9). (See Lemma 1 and Lemma 2 in Appendix B for details.)

Definition 2 An equilibrium is an allocation $\left\{\left(y_{i t}, h_{i t}, \overline{\mathfrak{m}}_{i t}, \mathfrak{m}_{i t+1}, \mathfrak{b}_{i t+1}\right)_{i \in \mathcal{I}}\right\}_{t=0}^{\infty}$, bargaining outcomes $\left\{\left(c_{i j t}\right)_{i, j \in \mathcal{I}}\right\}_{t=0}^{\infty}$, and pricing functions $\left\{\left(\phi_{t}(n)\right)_{n \in \overline{\mathcal{M}}_{t}},\left(q_{t}(s)\right)_{s \in \mathcal{B}_{t+1}}\right\}_{t=0}^{\infty}$, such that: (i) given prices and the bargaining protocol, $\left\{y_{i t}, h_{i t}, \overline{\mathfrak{m}}_{i t}, \mathfrak{m}_{i t+1}, \mathfrak{b}_{i t+1}\right\}_{t=0}^{\infty}$ solves agent $i$ 's optimization problem in the competitive markets for almost every $i \in \mathcal{I}$; (ii) for almost every $i, j \in \mathcal{I}$ in a bilateral meeting, the bilateral terms of trade are determined by Nash bargaining, i.e., if agent $i$ is the buyer and agent $j$ the seller in a bilateral meeting at time $t$, then $j$ produces $c_{i j t}=\min \left[\Lambda_{t}\left(\mathfrak{m}_{i t}, \mathfrak{b}_{i t}\right), c^{*}\right]$ special goods for $i\left(c_{i j t}=0\right.$ if $i$ and $j$ are not in a single-coincidence meeting at t); and (iii) the centralized market clears for all t. Let $\overline{\mathcal{M}}_{t}^{*}=\left\{n \in \overline{\mathcal{M}}_{t}: \phi_{t}(n)>0\right\}$, and $\mathcal{F}\left(\overline{\mathcal{M}}_{t}^{*}\right)=\left\{\mathcal{M} \in \mathcal{F}\left(\overline{\mathcal{M}}_{t}\right): \mathcal{M} \subseteq \overline{\mathcal{M}}_{t}^{*}\right\}$; an equilibrium is said to be "monetary" if $\overline{\mathcal{M}}_{t}^{*} \neq \varnothing$ for all $t$, and in this case, the allocation must also satisfy, for all $t$ : (a) the money-market clearing condition, $\int_{\mathcal{I}} \int_{\mathcal{M}} \overline{\mathfrak{m}}_{i t}(d n) \mu(d i)=\mu(\mathcal{M})$ for all $\mathcal{M} \in \mathcal{F}\left(\overline{\mathcal{M}}_{t}^{*}\right)$, and (b) the bond-market clearing condition, $\int_{\mathcal{I}} \int_{\mathcal{B}} \mathfrak{b}_{i t+1}(d s) \mu(d i)=\mu(\mathcal{B})$ for all $\mathcal{B} \in \mathcal{F}\left(\mathcal{B}_{t+1}\right)$. In a monetary equilibrium, money is said to coexist with bonds in period $t \geq 1$, if $B_{t}>0$ and $\int_{\mathcal{I}}\left(\phi_{t} \cdot \mathfrak{m}_{i t}\right) \mu(d i)>0$. A monetary equilibrium is said to be a "moneyspot equilibrium" if there exist $n, n^{\prime} \in \overline{\mathcal{M}}_{t}$ with $\phi_{t}(n) \neq \phi_{t}\left(n^{\prime}\right)$, for some $t$.

Proposition 2 Let $i_{t+1}(s)=1 / q_{t}(s)-1$ denote the nominal interest rate on a nominal bond with serial number $s \in \mathcal{B}_{t+1}$ between period $t$ and period $t+1$. If $u^{\prime}(0)>1+\frac{1-\beta}{\alpha \beta}$ and $\sup \mathbb{X}<\infty$, the economy with extraneous attributes admits a continuum of monetary equilibria in which money coexists with bonds for all $t<\infty$, even if $i_{t+1}(s)>0$ for all $s \in \mathcal{B}_{t+1}$.

The proof of Proposition 2 consists of constructing a family of moneyspot equilibria and showing that money coexists with bonds in any equilibrium that belongs to the family. The basic logic is as follows. If notes are distinguishable, e.g., by a serial number, then they need not be treated 
symmetrically by agents' beliefs, and different arbitrary subsets of the set of outstanding notes may be valued differently in equilibrium. The differences in valuations of extraneous attributes supported by these symmetry-breaking self-fulfilling beliefs can be used to construct rationalexpectations monetary equilibria with the property that fiat money coexists with interestbearing nominal bonds. To further illustrate the type of argument formalized in the proof of Proposition 2, consider the following streamlined example.

Suppose that in every period $t$, agents expect other agents to be willing to give up $\phi_{t}^{0}$ goods for notes whose serial numbers lie in some set $\overline{\mathcal{M}}_{t}^{0}$, and $\phi_{t}^{1}$ goods for notes whose serial numbers lie in another set $\overline{\mathcal{M}}_{t}^{1}$, where $\left\langle\overline{\mathcal{M}}_{t}^{0}, \overline{\mathcal{M}}_{t}^{1}\right\rangle$ is a partition of the set of time- $t$ post-redemption notes, $\overline{\mathcal{M}}_{t}$, and $\phi_{t}^{1}<\phi_{t}^{0}$. In addition, suppose that agents expect other agents to assign all notes injected by the government in period $t+s$ to the set $\overline{\mathcal{M}}_{t+s}^{1}$, and to value newly injected notes accordingly, at $\phi_{t+s}^{1}<\phi_{t+s}^{0}$. Agents' expectations are all that determine the sequence of partitions, $\left\{\left\langle\overline{\mathcal{M}}_{t}^{0}, \overline{\mathcal{M}}_{t}^{1}\right\rangle\right\}_{t=0}^{\infty}$, as well as the price differential between the notes. Intuitively, the content of Proposition 2 is that it is possible to construct equilibria in which all these expectations are mutually consistent and become self-fulfilling: In each period $t$, agents expect others to offer less goods for notes with serial numbers in $\overline{\mathcal{M}}_{t}^{1}$ than for notes with serial numbers in $\overline{\mathcal{M}}_{t}^{0}$, so they do so themselves, and since notes injected to redeem bonds at $t+1$ are expected to be worth $\phi_{t+1}^{1}<\phi_{t+1}^{0}$ general goods, agents are more inclined to purchase bonds with notes in $\overline{\mathcal{M}}_{t}^{1}$ than with notes in $\overline{\mathcal{M}}_{t}^{0}$. Given an expected price differential $\phi_{t+1}^{1} / \phi_{t+1}^{0}$ between both types of notes, it is possible for an open-market operation to be large enough to require bonds to be priced at a discount, but for this discount to be too small to induce agents to use notes in $\overline{\mathcal{M}}_{t}^{0}$ to purchase bonds that are risk-free in the usual sense that the government honors each bond with a note at maturity with probability one. In this case, an outside analyst would observe notes coexisting with nominal risk-free interest-bearing bonds.

\section{Moneyspots and the nominal interest rate}

An expectations-driven theory of nominal interest rate determination underlies the construction of moneyspot equilibria in the proof of Proposition 2. In this section, I discuss some implications of this theory for the behavior of the nominal interest rate. 


\subsection{Liquidity effects of open-market operations}

There are some similarities between the behavior of the nominal interest rate in moneyspot equilibria and in models with limited participation, e.g., Grossman and Weiss (1983), Lucas (1990), or Rotemberg (1984). Limited-participation models provide a theory of nominal interest rate determination with some attractive properties, such as the so-called "liquidity effects" of open-market operations. Those models rest on two fundamental assumptions that are critical for their ability to finesse the rate-of-return-dominance puzzle, and to generate these liquidity effects. First, by construction, they do not have to confront the rate-of-return-dominance puzzle: Those models are in the cash-in-advance tradition, in that money is assumed to be the only asset with which goods (and securities) can be bought. Second, the liquidity effects rely on specific timing assumptions as well as particular restrictions on portfolio reallocations.

In Lucas' version, for instance, there is a representative household composed of three members, each of whom carries out his own activity during a period, with the three regrouping at the end of the day to pool goods, assets, and information. One member collects the period endowment and sells it to other households on a cash-in-advance basis. ${ }^{16} \mathrm{~A}$ second member takes $N_{t}-Z_{t} \geq 0$ of the household's initial cash balances, $N_{t}$, and uses it to purchase goods from other households on a cash-in-advance basis. A third member of the household takes the remaining cash balances, $Z_{t}$, and engages in bond trading. A critical assumption is that the household commits itself to a division of cash among the three members before the size of the current period open-market operation is announced. Hence, there may sometimes be too much, and sometimes too little cash to be traded for bonds, which is the reason for the "liquidity effects." For example, the nominal rate will be low when the size of the open-market sale is large relative to the quantity of money that was committed in advance to the household member who participates in the bond market.

In Lucas' simplest formulation (e.g., Lucas, 1990, p. 242), the equilibrium price of bonds is $\min \left(z_{t}^{*} / x_{t}, 1\right)$, where $z_{t}^{*}$ is the proportion of the money supply that the representative household allocates to bond trading, and $x_{t}$ is, as in this paper, the size of the open-market operation relative to the outstanding money supply. In each of the equilibria that I construct in the proof of Proposition 2, the price of bonds is $\min \left(\left(1-\theta_{t}\right) / x_{t}, 1\right)$, where $1-\theta_{t} \equiv \mu\left(\overline{\mathcal{M}}_{t}^{1}\right) / \bar{M}_{t}$ is the proportion of the money supply that agents are willing to use for bond purchases. The expres-

\footnotetext{
${ }^{16}$ By assumption, a household cannot consume any of its own endowment, nor spend cash receipts from date- $t$ sales before date $t+1$.
} 
sion is identical to Lucas', with $1-\theta_{t}$ replacing $z_{t}^{*}$. The determination of the nominal interest rate in a moneyspot equilibrium is reminiscent of limited participation, but the mechanism is rather different: It is entirely driven by expectations and moneyspots, rather than by the specific timing and trading restrictions that the limited-participation formulation relies upon. ${ }^{17}$

\subsection{Negative nominal interest rates}

On occasion, nominal interest rates on government securities have become negative. For example, the transaction prices of Treasury Bills in the United States sometimes exceeded par during the Great Depression. The same happened with short-term Japanese Government bills late in 1998. The nominal interest rate on 4-week U.S. Treasury bills became negative twice in $2008 .{ }^{18}$ These instances are puzzling because, according to standard monetary theory, negative nominal interest rates are impossible given that agents can costlessly store money - an assumption that is implicitly adopted in most monetary models.

In this section I show that the nominal interest rate on government bonds can become negative in a moneyspot equilibrium, even if agents can store money costlessly. To this end, I consider a special case of the economy of Section 4 with a single open-market sale at $t=0$.

Proposition 3 Suppose that $B_{1}=x_{0} \bar{M}_{0}>0$, where $x_{0} \in \mathbb{X}$ is given at the beginning of period 0 , and $B_{t+1}=B_{0}=0$ for all $t \geq 1$. If $u^{\prime}(0)>1+\frac{1-\beta}{\alpha \beta}$ and $x_{0}<1$, the economy with extraneous attributes admits a continuum of monetary equilibria in which the nominal interest rate on government bonds is negative.

The proof of Proposition 3 consists of constructing a family of moneyspot equilibria and showing that the nominal interest rate between period 0 and period 1 is negative in any equilibrium that belongs to the family. The basic logic is as follows. Suppose that agents' expectations are that $\phi_{t}(n)=\phi_{t}^{s}$ for $n \in \overline{\mathcal{M}}_{t}^{s}$ and all $t$, for an expected sequence of partitions $\left\{\left\langle\overline{\mathcal{M}}_{t}^{0}, \overline{\mathcal{M}}_{t}^{1}\right\rangle\right\}_{t=0}^{\infty}$ with the property that for all $t \geq 1, \overline{\mathcal{M}}_{t}^{1}=\overline{\mathcal{M}}_{1}^{1}=\overline{\mathcal{M}}_{0}^{1} \cup \mathcal{N}_{1}^{+}$, where $\left\langle\overline{\mathcal{M}}_{0}^{0}, \overline{\mathcal{M}}_{0}^{1}\right\rangle$ is a partition

\footnotetext{
${ }^{17}$ The critical timing assumption is that money balances are allocated to the shopper and the financial trader before the size of the open-market operation shock is announced. The critical trading restriction is that money cannot be reallocated between the shopper and the financial trader once the size of the open market operation shock has been observed, e.g., in time to take advantage of the low bond prices if the open-market sale was large. There is no counterpart to either of these restrictions in this paper.

${ }^{18}$ The nominal rate on 4-week U.S. Treasury bills was negative on December 11 and December 19, 2008, see the Daily Treasury Bill Rates table published by the U.S. Treasury (U.S. Treasury, 2008). Cecchetti (1988) offers an account of negative nominal yields on U.S. Treasury bonds during the 1930s and early 1940s. WuDunn (1998) reports on Japan's negative rates in 1998.
} 
of $\overline{\mathcal{M}}_{0}$. In words, agents' expectations partition the set of outstanding notes at each date into notes of "type 0" and notes of "type 1." All the notes that are of type 1 in period 0 and all the notes that the government uses for bond redemptions in period 1, are regarded as notes of type 1 for all $t \geq 1$. Notice that according to these expectations, notes that are of type 0 in period 0 can become notes of type 1 in period 1 if the private sector uses them to purchase bonds in period 0 and the government uses them to redeem those bonds in period 1. (Notes that are regarded of type 0 in period 0 but are not used to purchase bonds in period 0 , remain of type 0 forever.) If in addition, agents expect $\phi_{t}^{1}=\phi_{1}^{1}>\phi_{t}^{0}=0$ for all $t \geq 1$, i.e., that notes of type 1 will remain in circulation while notes of type 0 will have no value from date 1 onwards, then at date 0 agents will be willing to use notes of type 0 to buy bonds at a premium. The proof of Proposition 3 shows that, for relatively small open-market sales at date 0 , there exist a continuum of moneyspot equilibria in which these expected partitions and prices are validated, and the nominal interest rate is negative.

\section{Final remarks}

I have shown that if the physical object that society uses as fiat money is heterogeneous in some extraneous attribute - a moneyspot - then there exist equilibria that induce outcomes that have traditionally proven difficult to rationalize, such as the coexistence of money and nominal riskfree interest-bearing government bonds, the liquidity effects of open-market operations, and the possibility of negative nominal interest rates. The formulation also articulates an expectationsdriven theory of the nominal interest rate, and illustrates the fact that, contrary to established wisdom, a positive nominal interest rate need not indicate a marketability advantage of money relative to bonds. From a theoretical standpoint, all this suggests that moneyspots can matter, and that they are worth studying.

At first, the idea that payoff-irrelevant attributes of money may be priced could seem odd. However, this is theoretically no different than having an intrinsically useless object being assigned a positive price, as is the case in any monetary equilibrium. In fact, whenever there is heterogeneity in the extraneous attributes of notes, the existence of equilibria in which moneyspots are priced would appear to be unavoidable in any good model of fiat money. ${ }^{19}$

\footnotetext{
${ }^{19}$ At some level, this point is no different from the "tenuousness" feature of monetary equilibria that Wallace (1977) deemed unavoidable in all good models of fiat money. This tenuousness alludes to the fact that a monetary equilibrium will typically coexist with a nonmonetary equilibrium. Wallace stresses that rather than being regarded as a defect, this tenuousness is natural, and can be helpful in interpreting some properties of
} 
An explicit model of extraneous monetary attributes requires making some specific modeling choices that have no counterpart in an ordinary model where the object that acts as money is assumed to be homogeneous. Let me enumerate what these modeling choices are, how they were made, and the ways in which they influence the main results.

First, in the formal modeling I have used serial numbers as the leading example of moneyspots, mainly because they have been one of the most universal features of paper money since its inception. As it was modeled above, this heterogeneity is very refined in that each note is effectively distinguishable from every other note. It should be evident from the construction in the proof of Proposition 2, that a version of the proposition can be established for a coarser specification of the heterogeneity in the extraneous attribute.

Second, to simplify the exposition, I have abstracted from the agents' decisions of whether to redeem a bond for a note. The working assumption has been that all bonds turn into notes at maturity (recall footnote 9 ). One could extend the model to explicitly incorporate an individual agent's redemption decision. I can see two natural ways to do this, and neither affects the results. One possibility is to assume that the government does not honor bonds that have exceeded their maturity, and does not accept matured bonds as payment for newly issued bonds. In this case there will be a monetary equilibrium in which matured bonds are not valued, and in such an equilibrium, all agents would be willing to redeem their bond holdings at maturity. The other possibility is to assume that the government stands ready to redeem any matured note. Then, in the class of equilibria constructed in the proof of Proposition 2, a matured bond would be priced just as a note of type 1 ; since agents would be indifferent between redeeming or not, it would be possible that matured bonds circulate alongside notes of type 0 , notes of type 1 , and unmatured bonds (with all bonds, and notes of type 1, trading at a discount relative to notes of type 0 ).

Third, I have assumed that the government ignores extraneous attributes, e.g., in the openmarket operation of time $t$, the government accepts any $q_{t}$ notes as payment for a newly issued bond (recall footnote 6 and constraint (8)). This symmetric government treatment of notes

monetary systems. Analogously, in the presence of extraneous (and arguably, realistic) characteristics of the object that will play the role of fiat money, monetary equilibria become even more tenuous, in the sense that the extraneous characteristics can coordinate expectations giving rise to a richer set of monetary equilibria. This higher degree of tenuousness is a natural feature of a model in which expectations are capable of rendering an intrinsically useless object valuable, and moreover, it sheds new light on some central issues in monetary theory. Also, the indeterminacy of the nominal interest rate in the equilibrium constructed in Proposition 2 is an unavoidable feature of the economy with moneyspots under laissez faire, much as the indeterminacy of the equilibrium exchange rate was an unavoidable feature in Kareken and Wallace (1981). 
makes it more difficult to support equilibria where notes are treated asymmetrically, which biases the theoretical model against the relevance of moneyspots. An explanation of rate-ofreturn dominance based on an exogenous government policy that favors some notes over others would have been much easier to formulate, but also more contrived and less revealing.

There is another aspect of government behavior that has been implicitly assumed, namely that it does not operate a "window" under a set of rules where agents can swap notes for notes. Can some arrangement of this type rule out the moneyspot equilibria of Proposition 2? After all, it is some well-run window of this sort what presumably keeps the exchange rate between one-dollar notes and ten-dollar notes fixed at ten-to-one. ${ }^{20}$ The answer is that even if the government in the model was ready to swap a note for another on demand, the moneyspot equilibria of Proposition 2 would still exist. The reason is that beliefs can be specified as in the proof of the proposition, so that any newly injected note (through window swaps, or bond redemptions) would be deemed of type 1 , and in any equilibrium in which these expectations are validated, the window would merely be swapping a note whose serial number belongs to the set of serial numbers of type 1 , for another note with a serial number in the same set. ${ }^{21}$

There have been instances in which money remained in circulation along with interestbearing, small-denomination, payable-to-the-bearer bonds issued by a government with the authority to print or tax the money needed to redeem those bonds. ${ }^{22}$ In light of impossibility results similar to Proposition 1, these instances have been regarded as puzzling - they embody

\footnotetext{
${ }^{20}$ Absent a well-functioning window, not even the fixed-exchange-rate regime among different denominations of a single currency can be taken for granted in monetary economies. For example, over the last couple of years in Argentina, coins have been privately trading at prices that exceed their face values against higher-denomination notes. The phenomenom appears to be generalized in some areas of the country, and has received a fair amount of press coverage (e.g., Galván, 2008, Surowiecki, 2009).

${ }^{21}$ This argument uses the fact that the heterogeneity in moneyspots is fine enough for each note to be distinguised from every other note. If notes contained just two extraneous attributes, e.g., if all notes were alike except that some were blue and the rest red, then the window could get rid of moneyspot equilibria in the same way that the Federal Reserve pegs exchange rates between notes of different denominations.

${ }^{22}$ Makinen and Woodward (1986) recount that small-denomination bearer bons issued by the government of France during 1915-27 seemed to circulate, at least to some extent, and exchanged only at a discount. Gherity (1993) provides examples of interest-bearing notes that circulated at a discount alongside non-ineterest-bearing notes (money) in the U.S. North during the Civil War (e.g., the Certificates of Indebtedness of 1862, the notes of 1863, the compound-interest notes of 1863, the seven-thirties of 1864 and 1865). Makinen and Woodward (1999) find evidence of the circulation of small-denomination bearer notes issued by the Confederate states during the Civil War. Wallace (1983, footnote 3) mentions the Liberty Bonds, which were issued by the United States during World War I, as small-denomination bearer securities that seem to have circulated as currency from time to time. More recently, several small-denomination bearer securities circulated extensively as currency alongside fiat money in Argentina between 2001 and 2003 (e.g., LECOPs, Patacones, etc.)
} 
the strongest version of the rate-of-return-dominance puzzle. ${ }^{23}$ As mentioned in the introduction, the basic observation goes back to Hicks's (1935) "suggestion." Since then, the issue has periodically caught the interest of monetary theorists.

In general, however, governments seldom issue bonds that literally fit the description in the impossibility propositions. ${ }^{24}$ In fact, an early argument for why fiat money consistently circulates even though there exist government-issued nominal securities that typically bear interest, is that the physical ways in which governments choose to materialize their nominal promises can cause marketability problems, e.g., due to large denominations with indivisibilities. In a series of well-known papers, Bryant and Wallace (1980) and Wallace (1983, 1990) advanced the hypothesis that fiat money is dominated in rate of return by government bonds in actual economies due to legal restrictions that prevent financial companies from intermediating the large-denomination bonds into securities or claims that are similar to money. There are a number of differences between moneyspots and legal restrictions as potential explanations of the coexistence puzzle. The main one is that according to the latter, absent legal and regulatory obstacles, interest-bearing bonds would be used in exchange, and there could not exist an equilibrium with non-interest-bearing notes. In contrast, moneyspot equilibria can rationalize coexistence even in the absence of legal restrictions. ${ }^{25}$

Abstract though it may seem, the rate-of-return-dominance puzzle has important implications for applied work. Impossibility results like Proposition 1 potentially subvert most of existing applied research in monetary economics, which by introducing outside money through shortcuts or reduced-form assumptions, take the coexistence of non-interest-bearing money and

\footnotetext{
${ }^{23}$ I call this the "strongest version" of the puzzle because an explanation is quite demanding, in that it calls for a theory that is able to rationalize why money is not driven out by bonds that pay interest, and have exactly the same payoff-relevant physical characteristics as money, e.g., issued in the same denominations, nominally risk-free, just as easy to exchange, just as costly to counterfeit, and so on. The observation that money is not driven out by interest-bearing risk-free bonds with different physical characteristics than money is in this sense a weaker version of the coexistence puzzle.

${ }^{24}$ In the United States, for instance, the Treasury issues bills that are payable to the bearer and nominally risk-free, but unlike U.S. Federal Reserve notes, they exist only as a book entry in a ledger at the Treasury. In the past, these bills existed in printed form, but the minimum denomination was rather large $(\$ 1,000)$.

${ }^{25}$ For instance, the examples in footnote 22 , which run counter to the legal restrictions hypothesis, can be rationalized as a moneyspot equilibrium. The legal restrictions hypothesis as it is usually stated does not seem compelling for the present-day United States. Since April 7, 2008 the minimum amount that can be purchased of any given U.S. Treasury bill, note, bond, or TIPS is $\$ 100$. A loose argument is often made, suggesting that contemporary bonds could not possibly be used as means of payment because they only exist as electronic book entries in a central electronic ledger. But whether ownership of the bond is manifested by a printed title or by a computer entry surely cannot matter. In fact, with the electronic trading systems that currently exist, e.g., the Commercial Book-Entry System (CBES), these book entries can be transferred pretty much instantaneously at a negligible technological cost, and the transfer could in principle be readily verified by the payer and the payee.
} 
higher-return assets as given. ${ }^{26}$ In models used to provide monetary policy recommendations, government-issued nominal bonds and fiat money are often assumed to coexist, with bonds trading at a discount. The implied nominal interest rate is analyzed, compared to data, used as a policy target, and so on. But given the impossibility propositions, how is it possible that the nominal rate is not identically zero at every date in those models? In models that assume money is an argument of a utility function, the answer is that it is assumed that outside money yields utility but bonds and inside money do not. In cash-in-advance models, the answer is that it is assumed that outside money satisfies the cash-in-advance constraint but bonds and inside money do not. In both cases the "answer" is a reduced-form modeling assumption. In contrast, as shown in Section 4, the nominal interest rate can be positive in a moneyspot equilibrium, even without assuming that money is intrinsically valuable, or that it can help agents overcome trading frictions in ways that bonds cannot.

Researchers who are mindful of the foundations of monetary exchange, a subset of whom tend to work on search models of money that satisfy the Wallace Dictum (e.g., Wallace, 1998), are well aware of the rate-of-return-dominance puzzle. In order to avoid internal inconsistencies and ad-hoc restrictions on portfolios or transaction patterns, they typically shun away from explicitly introducing nominal government bonds in their models. In order to introduce government-issued nominal bonds explicitly, some of these researchers impose explicit marketability restrictions that can be interpreted as versions of Wallace's legal restrictions. ${ }^{27}$ All in all, for the most part the search literature has not attempted to tackle the rate-of-returndominance puzzle. ${ }^{28}$

In modern economies, money (e.g., Federal Reserve notes) is held despite being dominated in rate of return by default-free claims to money (e.g., U.S. Treasury Bills). This is perhaps the most obvious instance of an asset pricing anomaly. This puzzle is central to monetary economics because a resolution requires a theory that explains the role that money is uniquely suited to play in actual economies, a role seemingly so specific that justifies foregoing the rate of return on assets that, through the lenses of our theories, ought to be regarded as equivalent to money. It seems to me that with the modern degree of financial and technological sophistication, the research agenda that consists of trying to uncover the frictions that explain why money is held

\footnotetext{
${ }^{26}$ Neil Wallace has been making this point for many years. See Wallace (1990, 1998), and Hellwig (1993).

${ }^{27}$ Andolfatto (2005), Berentsen and Waller (2008), Marchesiani and Senesi (2009), and Shi (2005), for example.

${ }^{28}$ There are two notable exceptions to this statement: Aiyagari, Wallace, and Wright (1996), and Zhu and Wallace (2007). In Appendix C, I review their main results and compare them with mine.
} 
despite being dominated in rate of return by default-free nominal bonds, is as challenging today as it was when Hicks wrote his "suggestion." I do not see a way of developing this agenda that does not begin by studying the set of equilibrium outcomes that can be implemented in the absence of these frictions, which is what I have tried to do in this paper. 


\section{A Proofs}

Proof of Proposition 1. The proof is organized in two parts. The first part shows that money cannot coexist with interest-bearing nominal bonds. The second part shows that the set of monetary equilibria is nonempty.

PART 1. The agent's problem in the bond market is

$$
U_{t}\left(\bar{m}_{t}\right)=\max _{0 \leq q_{t} b_{t+1} \leq \bar{m}_{t}} \beta E_{t} V_{t+1}\left(m_{t+1}, b_{t+1}\right),
$$

subject to $m_{t+1}=\bar{m}_{t}-q_{t} b_{t+1}$, and his problem in the general goods market is

$$
W_{t}\left(m_{t}, b_{t}\right)=\phi_{t}\left(m_{t}+b_{t}\right)+\max _{0 \leq \bar{m}_{t}}\left\{-\phi_{t} \bar{m}_{t}+U_{t}\left(\bar{m}_{t}\right)\right\}
$$

Combine these expressions to arrive at

$$
W_{t}\left(m_{t}, b_{t}\right)=\phi_{t}\left(m_{t}+b_{t}\right)+\max _{0 \leq \bar{m}_{t}}\left\{-\phi_{t} \bar{m}_{t}+\max _{0 \leq q_{t} b_{t+1} \leq \bar{m}_{t}} \beta E_{t} V_{t+1}\left(m_{t+1}, b_{t+1}\right)\right\},
$$

where $m_{t+1}=\bar{m}_{t}-q_{t} b_{t+1}$. Notice that $W_{t}\left(m_{t}, b_{t}\right)=\phi_{t}\left(m_{t}+b_{t}\right)+k_{t}$, where

$$
k_{t} \equiv \max _{0 \leq \bar{m}_{t}}\left\{-\phi_{t} \bar{m}_{t}+\max _{0 \leq q_{t} b_{t+1} \leq \bar{m}_{t}} \beta E_{t} V_{t+1}\left(\bar{m}_{t}-q_{t} b_{t+1}, b_{t+1}\right)\right\} \text {. }
$$

From (4),

$$
V_{t+1}\left(\bar{m}_{t}-q_{t} b_{t+1}, b_{t+1}\right)=\mathcal{L}\left(\phi_{t+1}\left[\bar{m}_{t}+\left(1-q_{t}\right) b_{t+1}\right]\right)+k_{t+1} .
$$

Thus, the agent's optimization problems in the centralized market and in the bond market are:

$$
\max _{0 \leq \bar{m}_{t}}\left\{-\phi_{t} \bar{m}_{t}+\max _{0 \leq q_{t} b_{t+1} \leq \bar{m}_{t}} \beta E_{t} \mathcal{L}\left(\phi_{t+1}\left[\bar{m}_{t}+\left(1-q_{t}\right) b_{t+1}\right]\right)\right\} .
$$

The solution to the inner maximization is:

$$
q_{t} b_{t+1} \begin{cases}=0 & \text { if } 1<q_{t} \\ \in\left[0, \bar{m}_{t}\right] & \text { if } q_{t}=1 \\ =\bar{m}_{t} & \text { if } q_{t}<1 .\end{cases}
$$

Notice that $q_{t}>1$ cannot be part of a monetary equilibrium, as it implies $\int_{\mathcal{I}} b_{i t+1} d \mu=0<B_{t+1}$, and the bond market would not clear. Hence, $q_{t} \leq 1$ for all $t$ in any monetary equilibrium. Suppose that $q_{t}<1$ at time $t$ in a monetary equilibrium. Then, from (14), every agent $i$ chooses $m_{i t+1}=0$ in the bond market at $t$, and therefore $\int_{\mathcal{I}} m_{i t+1} d \mu=0$, so money does not coexist with bonds in period $t+1$.

PART 2. In order to establish that the set of monetary equilibria of the economy with no extraneous attributes is nonempty, I construct a recursive monetary equilibrium for this economy. 
Definition 3 Let $\boldsymbol{s}_{t}=\left(x_{t}, \bar{M}_{t}\right)$ denote the aggregate state at time $t$. A recursive equilibrium of the economy with no extraneous attributes is a collection of individual decision rules $\bar{m}_{t}=\bar{m}\left(\boldsymbol{s}_{t}\right), m_{t+1}=m\left(\boldsymbol{s}_{t}\right), b_{t+1}=b\left(\boldsymbol{s}_{t}\right)$, together with bargaining outcomes $c_{t}=\mathrm{C}\left(\boldsymbol{s}_{t}\right)$, and pricing functions $\phi_{t}=\phi\left(s_{t}\right)$ and $q_{t}=q\left(s_{t}\right)$, such that: (i) given prices and the bargaining protocol, the decision rules $\bar{m}(\cdot), m(\cdot)$, and $b(\cdot)$, solve the agent's problem in the centralized markets; (ii) the bilateral terms of trade are determined by Nash bargaining, i.e., $\mathrm{C}\left(\boldsymbol{s}_{t}\right)=\min \left[\phi\left(\boldsymbol{s}_{t}\right) \bar{m}\left(\boldsymbol{s}_{t}\right), c^{*}\right]$; and (iii) prices are such that centralized markets clear. The equilibrium is "monetary" if $\phi\left(\boldsymbol{s}_{t}\right)>0$ for all $\boldsymbol{s}_{t}$, and in this case the money-market clearing condition $\bar{m}\left(s_{t}\right)=\bar{M}_{t}$, and the bond-market clearing condition is $b\left(s_{t}\right)=x_{t} \bar{M}_{t}$ must hold.

Given (14), the agent's problem in the centralized market before the bond issue is

$$
\max _{0 \leq \bar{m}_{t}}\left\{-\phi_{t} \bar{m}_{t}+\beta E_{t} \mathcal{L}\left(\phi_{t+1}\left[\bar{m}_{t}+\left(1-q_{t}\right) g_{t}\left(\bar{m}_{t}\right)\right]\right)\right\}
$$

where

$$
g_{t}\left(\bar{m}_{t}\right) \begin{cases}=0 & \text { if } 1<q_{t} \\ \in\left[0, \frac{\bar{m}_{t}}{q_{t}}\right] & \text { if } q_{t}=1 \\ =\frac{\bar{m}_{t}}{q_{t}} & \text { if } q_{t}<1\end{cases}
$$

Since

$$
\bar{m}_{t}+\left(1-q_{t}\right) g_{t}\left(\bar{m}_{t}\right)=\max \left(\frac{1}{q_{t}}, 1\right) \bar{m}_{t}
$$

this problem can be written as

$$
\max _{0 \leq \bar{m}_{t}}\left\{-\phi_{t} \bar{m}_{t}+\beta E_{t} \mathcal{L}\left[\max \left(\frac{1}{q_{t}}, 1\right) \phi_{t+1} \bar{m}_{t}\right]\right\}
$$

and the corresponding first-order condition is

$$
-\phi_{t}+\beta E_{t}\left\{\mathcal{L}^{\prime}\left[\max \left(\frac{1}{q_{t}}, 1\right) \phi_{t+1} \bar{m}_{t}\right] \max \left(\frac{1}{q_{t}}, 1\right) \phi_{t+1}\right\} \leq 0, \quad \text { "= " if } \bar{m}_{t}>0 .
$$

In a monetary equilibrium, $\bar{m}_{t}=\bar{M}_{t}>0$ (money-market clearing), and $g_{t}\left(\bar{M}_{t}\right)=B_{t+1}$ (bondmarket clearing), so (15) implies

$$
\bar{M}_{t+1}=\max \left(\frac{1}{q_{t}}, 1\right) \bar{M}_{t}
$$

Thus in a monetary equilibrium, (16) together with (17) give the Euler equation

$$
\phi_{t} \bar{M}_{t}=\beta E_{t}\left[\mathcal{L}^{\prime}\left(\phi_{t+1} \bar{M}_{t+1}\right) \phi_{t+1} \bar{M}_{t+1}\right] .
$$


The equilibrium conditions $\bar{m}_{t}=\bar{M}_{t}$ and $b_{t+1}=B_{t+1}$ and (14) imply $q_{t}=\min \left(\bar{M}_{t} / B_{t+1}, 1\right)$. Since $B_{t+1}=x_{t} \bar{M}_{t}$, the equilibrium price of bonds is

$$
q_{t}=\min \left(x_{t}^{-1}, 1\right)
$$

and the nominal interest rate is

$$
i_{t+1}=\max \left(x_{t}-1,0\right)
$$

Given (17) and (19), the post-redemption stock of notes evolves according to

$$
\bar{M}_{t+1}=\max \left(x_{t}, 1\right) \bar{M}_{t}
$$

in a monetary equilibrium. The quantity of money that agents carry out of the bond market into the search market is

$$
M_{t+1}=\max \left(1-x_{t}, 0\right) \bar{M}_{t}
$$

Define real money balances, $z_{t} \equiv \phi_{t} \bar{M}_{t}$, and look for a recursive equilibrium where $z_{t}=z\left(x_{t}\right) \equiv$ $\phi\left(s_{t}\right) \bar{M}_{t}$. Then the Euler equation (18) becomes

$$
z(x)=\beta \int \mathcal{L}^{\prime}\left[z\left(x^{\prime}\right)\right] z\left(x^{\prime}\right) d F\left(x^{\prime}, x\right) .
$$

Given a positive $z(\cdot)$ that solves $(21)$, a recursive monetary equilibrium is then an allocation $\bar{m}\left(s_{t}\right)=\bar{M}_{t}, b\left(s_{t}\right)=x_{t} \bar{M}_{t}, m\left(s_{t}\right)=\max \left[\left(1-x_{t}\right) \bar{M}_{t}, 0\right]$, bargaining outcomes $\mathrm{C}\left(x_{t}\right)=$ $\min \left[z\left(x_{t}\right), c^{*}\right]$, together with the pricing function for notes, $\phi\left(s_{t}\right)=z\left(x_{t}\right) / \bar{M}_{t}$ and the pricing function for bonds, $q\left(x_{t}\right)=\min \left(x_{t}^{-1}, 1\right)$. Notice that a solution to $(21)$ is $z(x)=z$ for all $x$, where $z \in\left(0, c^{*}\right)$ is the unique solution to $\beta \mathcal{L}^{\prime}(z)=1$.

Proof of Proposition 2. The proof is organized in three parts. Part 1 derives the set of equilibrium conditions that characterize a monetary equilibrium that belongs to a certain class. Part 2 constructs a particular allocation and price system, and establishes that they constitute a family (a continuum) of monetary equilibria that belong to the class described in Part 1. Part 3 establishes that in the family of monetary equilibria described in Part 2, money coexists with bonds for all $t$, even if $q_{t}(s)<1$ for all $s \in \mathcal{B}_{t+1}$.

PART 1. Focus on a class of monetary equilibria in which agents' beliefs endogenously partition the set of outstanding notes into two subsets such that any two notes are treated identically if 
and only if their serial numbers belong to the same subset. Let $\left\langle\mathcal{M}_{t}^{0}, \mathcal{M}_{t}^{1}\right\rangle$ be a partition of the set of beginning-of-period- $t$ outstanding notes, $\mathcal{M}_{t}$, and let $\left\langle\mathcal{N}_{t}^{0+}, \mathcal{N}_{t}^{1+}\right\rangle$ be a partition of the set of notes injected in period $t, \mathcal{N}_{t}^{+}$. Given $\left\langle\mathcal{M}_{t}^{0}, \mathcal{M}_{t}^{1}\right\rangle$ and $\left\langle\mathcal{N}_{t}^{0+}, \mathcal{N}_{t}^{1+}\right\rangle$, posit that the post-redemption set of notes of type $s=0,1$, is $\overline{\mathcal{M}}_{t}^{s}=\mathcal{M}_{t}^{s} \cup \mathcal{N}_{t}^{s+}$. (Hereafter, I will refer to notes whose serial numbers lie in $\mathcal{M}_{t}^{s}, \mathcal{N}_{t}^{s+}$, or $\overline{\mathcal{M}}_{t}^{s}$, as notes of type s.) At the end of period $t$, the government issues a set of bonds $\mathcal{B}_{t+1}$ of size $B_{t+1}=\mu\left(\mathcal{B}_{t+1}\right)$, thereby withdrawing a set of notes $\mathcal{N}_{t+1}^{-}$. The set of notes withdrawn in period $t, \mathcal{N}_{t+1}^{-}$, is determined by the equilibrium trades in the bond market along with the partition $\left\langle\mathcal{N}_{t+1}^{0-}, \mathcal{N}_{t+1}^{1-}\right\rangle$, where $\mathcal{N}_{t+1}^{s-}=\mathcal{N}_{t+1}^{-} \cap \overline{\mathcal{M}}_{t}^{s}$ represents the set of notes of type $s$ withdrawn by the bond issue of period $t$. Next, I obtain the set of equilibrium conditions that characterize a monetary equilibrium within a class with certain properties.

Property 1. At $t=0$, agents' beliefs are that

$$
\mathcal{M}_{0}^{0}=\left[0, \theta_{0} M_{0}\right] \quad \text { and } \quad \mathcal{M}_{0}^{1}=\left(\theta_{0} M_{0}, M_{0}\right]
$$

where $\theta_{0}$ is an arbitrary number in the interval $(0,1)$. For $t \geq 0$, agents' beliefs specify

$$
\mathcal{N}_{t}^{0+}=\varnothing
$$

for newly injected notes, and

$$
\overline{\mathcal{M}}_{t+1}^{s}=\left(\overline{\mathcal{M}}_{t}^{s \backslash} \backslash \mathcal{N}_{t+1}^{s-}\right) \cup \mathcal{N}_{t+1}^{s+}
$$

for the law of motion of the post-redemption set of outstanding notes of type $s$.

Property 2. For $s=0,1$ and every $t, \phi_{t}(n)=\phi_{t}^{s}>0, \forall n \in \overline{\mathcal{M}}_{t}^{s}$.

An implication of Property 2 is that $\overline{\mathcal{M}}_{t}^{*}=\overline{\mathcal{M}}_{t}$ for every $t$. With regards to Property 1 , notice that (23) implies $\overline{\mathcal{M}}_{0}^{0}=\mathcal{M}_{0}^{0}$, and that all bonds have the same probability of being redeemed by a note of type $s$ (this probability is 1 for $s=1$, and 0 for $s=0$ ), which makes each bond effectively identical to every other bond. Since every bond is worth the same to private agents regardless of the serial number printed on it, competitive trade at the time of issue implies $q_{t}(s)=q_{t}$ for all $s \in \mathcal{B}_{t+1}$. With this, I can write

$$
\mathcal{N}_{t+1}^{1+}=\mathcal{N}_{t+1}^{+}= \begin{cases}\mathcal{N}_{t+1}^{-} \cup\left(\bar{M}_{t},\left[1+\left(1-q_{t}\right) x_{t}\right] \bar{M}_{t}\right] & \text { if } q_{t} \leq 1 \\ \mathcal{N}_{t+1}^{-} \cap\left[0, \eta_{t}\left(x_{t} \bar{M}_{t}\right)\right] & \text { if } q_{t}>1\end{cases}
$$


for $t \geq 0$, where $\eta_{t}(z) \equiv \inf \left\{n \in \overline{\mathcal{M}}_{t} \mid \mu\left(\mathcal{N}_{t+1}^{-} \cap[0, n]\right)=z\right\}$.

Let $M_{t}^{s}=\mu\left(\mathcal{M}_{t}^{s}\right), \bar{M}_{t}^{s}=\mu\left(\overline{\mathcal{M}}_{t}^{s}\right)$, and for $q_{t}>0$, let $B_{t+1}^{s}=\mu\left(\mathcal{N}_{t+1}^{s-}\right) / q_{t}$. Intuitively, $M_{t}^{s}$ represents the quantity of notes of type $s$ outstanding at the beginning of period $t, \bar{M}_{t}^{s}$ represents the quantity of notes of type $s$ outstanding after the period- $t$ bond redemption, and $B_{t+1}^{s}$ is the measure of bonds issued in period $t$ that are purchased with notes of type $s$. The law of motion (24) implies

$$
\bar{M}_{t+1}^{s}=\bar{M}_{t}^{s}-q_{t} B_{t+1}^{s}+\mu\left(\mathcal{N}_{t+1}^{s+}\right)
$$

for $s=0,1$, and together, the two conditions in (25) imply (1).

Next, I derive two implications of Properties 1 and 2. Agent $i$ 's problem in the bond market at time $t$ (the inner maximization in (13)) only depends on the portfolio of assets being chosen, $\mathfrak{a}_{i t+1}$, through $\Lambda_{t+1}\left(\mathfrak{a}_{i t+1}\right)$. Given Properties 1 and 2 ,

$$
\Lambda_{t+1}\left(\mathfrak{a}_{i t+1}\right)=\phi_{t+1} \boldsymbol{m}_{i t+1}+\phi_{t+1}^{1} b_{i t+1}
$$

where $\phi_{t+1} \equiv\left(\phi_{t+1}^{0}, \phi_{t+1}^{1}\right), \boldsymbol{m}_{i t+1} \equiv\left(m_{i t+1}^{0}, m_{i t+1}^{1}\right)$ with $m_{i t+1}^{s} \equiv \mathfrak{m}_{i t+1}\left(\overline{\mathcal{M}}_{t}^{s}\right)$, and $b_{i t+1} \equiv$ $\mathfrak{b}_{i t+1}\left(\mathcal{B}_{t+1}\right)=\mathfrak{n}_{i t+1}^{+}\left(\mathcal{N}_{t+1}^{+}\right)$. This leads to the first implication: the agent's problem in the bond market consists of choosing the quantity of bonds to buy from the government, and the quantities of notes of each type to use as payment. The relevant notation is as follows. The measure of notes of type $s$ that agent $i$ exchanges for bonds at time $t$ is $n_{i t+1}^{s-} \equiv \mathfrak{n}_{i t+1}^{-}\left(\overline{\mathcal{M}}_{t}^{s}\right)$, while $n_{i t+1}^{-} \equiv \mathfrak{n}_{i t+1}^{-}\left(\overline{\mathcal{M}}_{t}\right)=n_{i t+1}^{0-}+n_{i t+1}^{1-}$ is the total quantity of notes that $i$ uses to buy bonds at $t$. For $q_{t}>0, b_{i t+1}^{s} \equiv n_{i t+1}^{s-} / q_{t}$ denotes the measure of bonds that agent $i$ chooses to buy with notes of type $s$. It is also convenient to define $\boldsymbol{b}_{i t+1} \equiv\left(b_{i t+1}^{0}, b_{i t+1}^{1}\right)$, where $b_{i t+1}^{0}+b_{i t+1}^{1}$ is the total quantity of bonds purchased by agent $i$, i.e., $b_{i t+1}^{0}+b_{i t+1}^{1}=b_{i t+1}$. The second useful implication of Properties 1 and 2 is that the objective that agent $i$ seeks to maximize in the centralized market at time $t$ (the outer maximization in (13)), only depends on $\overline{\mathfrak{m}}_{i t}$ through $\phi_{t} \cdot \overline{\mathfrak{m}}_{i t}$, and that $\phi_{t} \cdot \overline{\mathfrak{m}}_{i t}=\phi_{t} \overline{\boldsymbol{m}}_{i t}$, where $\overline{\boldsymbol{m}}_{i t} \equiv\left(\bar{m}_{i t}^{0}, \bar{m}_{i t}^{1}\right)$, with $\bar{m}_{i t}^{s} \equiv \overline{\mathfrak{m}}_{i t}\left(\overline{\mathcal{M}}_{t}^{s}\right)$.

Lemma 2 (see Appendix B) uses the two previous implications of Properties 1 and 2 to establish that in a monetary equilibrium in which $\left\{\left\langle\overline{\mathcal{M}}_{t+1}^{0}, \overline{\mathcal{M}}_{t+1}^{1}\right\rangle\right\}_{t=0}^{\infty}$ and $\left\{\phi_{t}\right\}_{t=0}^{\infty}$ satisfy Properties 1 and 2, a solution to agent $i$ 's time- $t$ optimization problem, (13), is a collection of measures $\left(\overline{\mathfrak{m}}_{i t}, \mathfrak{m}_{i t+1}, \mathfrak{b}_{i t+1}, \mathfrak{n}_{i t+1}^{-}\right)$that satisfy $\overline{\mathfrak{m}}_{i t} \in \overline{\mathfrak{M}}_{t},(7)$ with equality and (9), such that the associated quantities $\left(\bar{m}_{i t}^{s}, m_{i t+1}^{s}, b_{i t+1}^{s}, n_{i t+1}^{s-}\right)_{s=0,1}$ achieve

$$
\Delta_{t}=\max _{\overline{\boldsymbol{m}}_{i t} \in \mathbb{R}^{+} \times \mathbb{R}^{+}}\left\{-\boldsymbol{\phi}_{t} \overline{\boldsymbol{m}}_{i t}+\max _{\mathbf{0} \leq q_{t} \boldsymbol{b}_{i t+1} \leq \overline{\boldsymbol{m}}_{i t}} \beta E_{t} \mathcal{L}\left[\lambda_{t+1}\left(\overline{\boldsymbol{m}}_{i t}, \boldsymbol{b}_{i t+1}\right)\right]\right\}
$$


with

$$
m_{i t+1}^{s}=\bar{m}_{i t}^{s}-q_{t} b_{i t+1}^{s}
$$

and $n_{i t+1}^{s-}=q_{t} b_{i t+1}^{s}$ for $s=0,1$, and with $\lambda_{t+1}: \mathbb{R}^{4} \rightarrow \mathbb{R}$ defined by

$$
\lambda_{t+1}\left(\overline{\boldsymbol{m}}_{i t}, \boldsymbol{b}_{i t+1}\right)=\sum_{s=0}^{1}\left[\phi_{t+1}^{s} \bar{m}_{i t}^{s}+\left(\bar{q}_{t}^{s}-q_{t}\right) \phi_{t+1}^{s} b_{i t+1}^{s}\right],
$$

where $\bar{q}_{t}^{s} \equiv \phi_{t+1}^{1} / \phi_{t+1}^{s}$.

Property 3. The sequence $\left\{\phi_{t}^{0}, \phi_{t}^{1}\right\}_{t=0}^{\infty}$ is such that $\phi_{t+1}^{s}$ is known at time $t$.

If Property 3 holds, the solution to the maximization problem of an agent $i$ who enters the market for bonds at time $t$ carrying a vector $\overline{\boldsymbol{m}}_{i t}$ of notes (the inner maximization on the right side of $(26))$ is $b_{i t+1}^{s}=g_{t}^{s}\left(\bar{m}_{i t}^{s}\right)$, where

$$
g_{t}^{s}\left(\bar{m}_{i t}^{s}\right) \begin{cases}=0 & \text { if } \bar{q}_{t}^{s}<q_{t} \\ \in\left[0, \frac{\bar{m}_{i t}^{s}}{q_{t}}\right] & \text { if } q_{t}=\bar{q}_{t}^{s} \\ =\frac{\bar{m}_{i t}^{s}}{q_{t}} & \text { if } q_{t}<\bar{q}_{t}^{s} .\end{cases}
$$

With (28), agent $i$ 's problem in the centralized market of period $t$ (the outer maximization on the right side of (26)) becomes

$$
\max _{\overline{\boldsymbol{m}}_{i t} \in \mathbb{R}^{+} \times \mathbb{R}^{+}}\left\{-\boldsymbol{\phi}_{t} \overline{\boldsymbol{m}}_{i t}+\beta E_{t} \mathcal{L}\left[\lambda_{t+1}^{*}\left(\overline{\boldsymbol{m}}_{i t}\right)\right]\right\}
$$

where

$$
\begin{aligned}
\lambda_{t+1}^{*}\left(\overline{\boldsymbol{m}}_{i t}\right) & =\sum_{s=0}^{1}\left[\phi_{t+1}^{s} \bar{m}_{i t}^{s}+\left(\bar{q}_{t}^{s}-q_{t}\right) \phi_{t+1}^{s} g_{t}^{s}\left(\bar{m}_{i t}^{s}\right)\right] \\
& =\sum_{s=0}^{1} \max \left(\frac{\bar{q}_{t}^{s}}{q_{t}}, 1\right) \phi_{t+1}^{s} \bar{m}_{i t}^{s} .
\end{aligned}
$$

With Property 3 , it is easy to check that the objective, $-\boldsymbol{\phi}_{t} \overline{\boldsymbol{m}}_{i t}+\beta E_{t} \mathcal{L}\left[\lambda_{t+1}^{*}\left(\overline{\boldsymbol{m}}_{i t}\right)\right]$, is a concave function of $\overline{\boldsymbol{m}}_{i t}$, so the following first-order conditions are necessary and sufficient for an optimum of the agent's problem in the centralized market of period $t$

$$
-\phi_{t}^{s}+\beta E_{t}\left\{\mathcal{L}^{\prime}\left[\lambda_{t+1}^{*}\left(\overline{\boldsymbol{m}}_{i t}\right)\right] \max \left(\frac{\bar{q}_{t}^{s}}{q_{t}}, 1\right) \phi_{t+1}^{s}\right\} \leq 0, \quad "=" \text { if } \bar{m}_{i t}^{s}>0, \quad \text { for } s=0,1 .
$$

Property 4. $\quad \overline{\boldsymbol{m}}_{i t}=\overline{\boldsymbol{m}}_{t}$ for a.e. $i \in \mathcal{I}$. 
The money-market clearing condition is $\int_{\mathcal{I}} \int_{\mathcal{M}} \overline{\mathfrak{m}}_{i t}(d n) \mu(d i)=\mu(\mathcal{M})$ for all $\mathcal{M} \in \mathcal{F}\left(\overline{\mathcal{M}}_{t}\right)$. Evaluate it at $\mathcal{M}=\overline{\mathcal{M}}_{t}^{s}$ to get $\int_{\mathcal{I}} \bar{m}_{i t}^{s} \mu(d i)=\bar{M}_{t}^{s}$, which combined with Property 4 implies $\overline{\boldsymbol{m}}_{t}=\left(\bar{M}_{t}^{0}, \bar{M}_{t}^{1}\right) \equiv \overline{\boldsymbol{M}}_{t}$. Together with (28), this implies $g_{t}^{s}\left(\bar{m}_{i t}^{s}\right)=g_{t}^{s}\left(\bar{M}_{t}^{s}\right)$, so from (27), $m_{i t+1}^{s}=\bar{M}_{t}^{s}-q_{t} g_{t}^{s}\left(\bar{M}_{t}^{s}\right)$, so $\boldsymbol{m}_{i t+1}=\left(M_{t+1}^{0}, M_{t+1}^{1}\right) \equiv \boldsymbol{M}_{t+1}$. Therefore, $q_{t} g_{t}^{s}\left(\bar{M}_{t}^{s}\right)=\bar{M}_{t}^{s}-$ $M_{t+1}^{s}$, which implies that $q_{t} g_{t}^{s}\left(\bar{M}_{t}^{s}\right)=\mu\left(\mathcal{N}_{t+1}^{s-}\right)$, but since $q_{t} B_{t+1}^{s} \equiv \mu\left(\mathcal{N}_{t+1}^{s-}\right)$, it follows that $g_{t}^{s}\left(\bar{M}_{t}^{s}\right)=B_{t+1}^{s}$ for $s=0,1$. In summary, Property 4 implies that, in equilibrium, $\bar{m}_{i t}^{s}=\bar{M}_{t}^{s}$, $m_{i t+1}^{s}=M_{t+1}^{s}$, and $b_{i t+1}^{s}=B_{t+1}^{s}$ for $s=0,1$. Since (22), (23), (24), (25) and (29) imply

$$
\lambda_{t+1}^{*}\left(\bar{M}_{t}\right)=\phi_{t+1} \bar{M}_{t+1} \equiv z_{t+1}
$$

in a monetary equilibrium in which both types of notes are held, (31) becomes

$$
\phi_{t}^{s}=\beta E_{t}\left[\mathcal{L}^{\prime}\left(z_{t+1}\right) \max \left(\frac{\bar{q}_{t}^{s}}{q_{t}}, 1\right) \phi_{t+1}^{s}\right], \quad \text { for } s=0,1 .
$$

Also, (22), (23), (24), (25) and (28) yield

$$
\begin{aligned}
& \bar{M}_{t+1}^{0}=\left[1-\frac{g_{t}^{0}\left(\bar{M}_{t}^{0}\right)}{\bar{M}_{t}^{0}} q_{t}\right] \bar{M}_{t}^{0} \\
& \bar{M}_{t+1}^{1}=\left[\max \left(\frac{1}{q_{t}}, 1\right)+\frac{g_{t}^{0}\left(\bar{M}_{t}^{0}\right)}{\bar{M}_{t}^{1}}\right] \bar{M}_{t}^{1},
\end{aligned}
$$

so with $z_{t}^{s} \equiv \phi_{t}^{s} \bar{M}_{t}^{s}$ for $s=0,1,(32)$ can be written as

$$
\begin{aligned}
z_{t}^{0} & =\frac{\max \left(\frac{\bar{q}_{t}^{0}}{q_{t}}, 1\right)}{1-\frac{g_{t}^{0}\left(\bar{M}_{t}^{0}\right)}{M_{t}^{0}} q_{t}} \beta E_{t}\left[\mathcal{L}^{\prime}\left(z_{t+1}\right) z_{t+1}^{0}\right] \\
z_{t}^{1} & =\frac{1}{1+\frac{g_{t}^{0}\left(\bar{M}_{t}^{0}\right)}{\max \left(\frac{1}{q_{t}}, 1\right) \bar{M}_{t}^{1}}} \beta E_{t}\left[\mathcal{L}^{\prime}\left(z_{t+1}\right) z_{t+1}^{1}\right] .
\end{aligned}
$$

The bond-market clearing condition requires

$$
B_{t+1}^{0}+B_{t+1}^{1}=B_{t+1}
$$

The supply of bonds at time $t$ is $B_{t+1}=x_{t} \bar{M}_{t}$. With $g_{t}^{s}\left(\bar{M}_{t}^{s}\right)=B_{t+1}^{s}$ and (28), the aggregate 
demand for bonds, $G_{t}\left(\overline{\boldsymbol{M}}_{t}\right) \equiv \sum_{s=0}^{1} g_{t}^{s}\left(\bar{M}_{t}^{s}\right)$ is

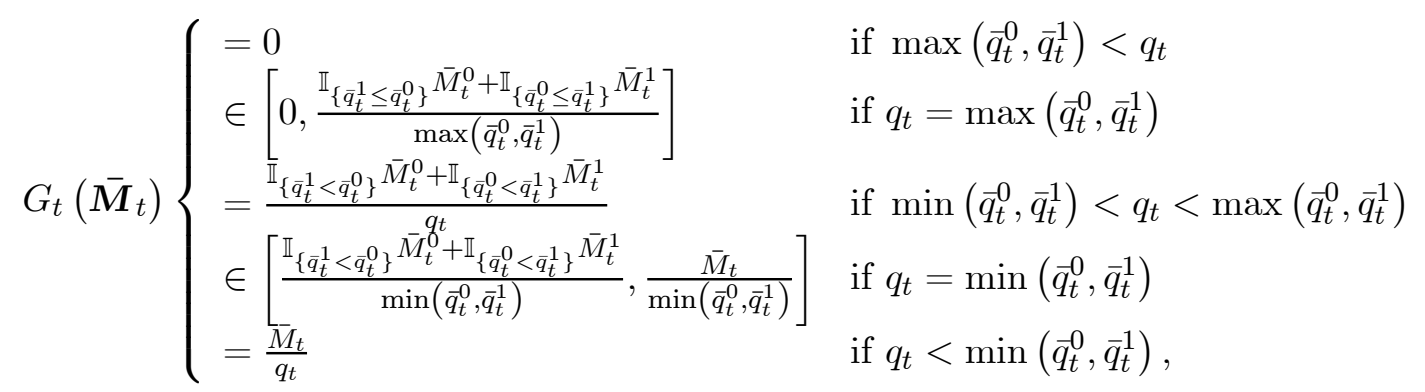

where $\mathbb{I}_{\left\{\bar{q}_{t}^{1} \leq \bar{q}_{t}^{0}\right\}}$ is an indicator function that takes the value 1 if $\bar{q}_{t}^{1} \leq \bar{q}_{t}^{0}$. Hence (37) can be written as $G_{t}\left(\overline{\boldsymbol{M}}_{t}\right)=x_{t} \bar{M}_{t}$, which implies that the equilibrium price of bonds satisfies

$$
q_{t}= \begin{cases}\max \left(\bar{q}_{t}^{0}, \bar{q}_{t}^{1}\right) & \text { if } x_{t} \leq \frac{\mathbb{I}_{\left\{\bar{q}_{t}^{1} \leq \bar{q}_{t}^{0}\right\}} \theta_{t}+\mathbb{I}_{\left\{\bar{q}_{t}^{0} \leq \bar{q}_{t}^{1}\right\}}\left(1-\theta_{t}\right)}{\max \left(\bar{q}_{t}^{0}, \bar{q}_{t}^{1}\right)} \\ \frac{\mathbb{I}_{\left\{\bar{q}_{t}^{1}<\bar{q}_{t}^{0}\right\}} \theta_{t}+\mathbb{I}_{\left\{\bar{q}_{t}^{0}<\bar{q}_{t}^{1}\right\}}\left(1-\theta_{t}\right)}{x_{t}} & \text { if } \frac{\mathbb{I}_{\left\{\bar{q}_{t}^{1} \leq \bar{q}_{t}^{0}\right\}} \theta_{t}+\mathbb{I}_{\left\{\bar{q}_{t}^{0} \leq \bar{q}_{t}^{1}\right\}}\left(1-\theta_{t}\right)}{\max \left(\bar{q}_{t}^{0}, \bar{q}_{t}^{1}\right)}<x_{t}<\frac{\mathbb{I}_{\left\{\bar{q}_{t}^{1}<\bar{q}_{t}^{0}\right\}} \theta_{t}+\mathbb{I}_{\left\{\bar{q}_{t}^{0}<\bar{q}_{t}^{1}\right\}}\left(1-\theta_{t}\right)}{\min \left(\bar{q}_{t}^{0}, \bar{q}_{t}^{1}\right)} \\ \min \left(\bar{q}_{t}^{0}, \bar{q}_{t}^{1}\right) & \text { if } \frac{\mathbb{I}_{\left\{\bar{q}_{t}^{1}<\bar{q}_{t}^{0}\right\}} \theta_{t}+\mathbb{I}_{\left\{\bar{q}_{t}^{0}<\bar{q}_{t}^{1}\right\}}\left(1-\theta_{t}\right)}{\min \left(\bar{q}_{t}^{0}, \bar{q}_{t}^{1}\right)} \leq x_{t} \leq \frac{1}{\min \left(\bar{q}_{t}^{0}, \bar{q}_{t}^{1}\right)} \\ \frac{1}{x_{t}} & \text { if } \frac{1}{\min \left(\bar{q}_{t}^{0}, \bar{q}_{t}^{1}\right)}<x_{t},\end{cases}
$$

where $\theta_{t} \equiv \bar{M}_{t}^{0} / \bar{M}_{t}$. Finally, the bond-market constraint (27) implies

$$
\boldsymbol{M}_{t+1}=\overline{\boldsymbol{M}}_{t}-q_{t} \boldsymbol{B}_{t+1},
$$

where $\boldsymbol{B}_{t+1}=\left(B_{t+1}^{0}, B_{t+1}^{1}\right)$.

In summary, a monetary equilibrium that satisfies Properties 1-4 consists of an allocation $\left\{\left(y_{i t}, h_{i t}, \overline{\mathfrak{m}}_{i t}, \mathfrak{m}_{i t+1}, \mathfrak{b}_{i t+1}, \mathfrak{n}_{i t+1}^{-}\right)_{i \in \mathcal{I}}\right\}_{t=0}^{\infty}$, prices $\left\{\left(\phi_{t}(n)\right)_{n \in \overline{\mathcal{M}}_{t}},\left(q_{t}(s)\right)_{s \in \mathcal{B}_{t+1}}\right\}_{t=0}^{\infty}$, and bargaining outcomes, $\left\{\left(c_{i j t}\right)_{i, j \in \mathcal{I}}\right\}_{t=0}^{\infty}$, such that:

(E1). $\quad c_{i j t}=\min \left(z_{t}, c^{*}\right) \mathbb{I}_{\left\{i \in \mathcal{I}_{t}^{b}\right\}}(i)$ for a.e. $i, j \in \mathcal{I}$, where $\mathbb{I}_{\{i \in \mathcal{A}\}}(i)$ is an indicator function that takes the value 1 if $i \in \mathcal{A}$.

(E2). $y_{i t}$ and $h_{i t}$, satisfy $h_{i t}=y_{i t}+\phi_{t} \overline{\boldsymbol{M}}_{t} \mathbb{I}_{\left\{i \in \mathcal{I}_{t}^{b}\right\}}(i)-\phi_{t} \overline{\boldsymbol{M}}_{t} \mathbb{I}_{\left\{i \in \mathcal{I}_{t}^{s}\right\}}(i)$, with $0 \leq y_{i t}$, and $0 \leq h_{i t} \leq \bar{h}$, for a.e. $i \in \mathcal{I}$.

(E3). $q_{t}(s)=q_{t}$ for all $s \in \mathcal{B}_{t+1}$, with $q_{t}$ given by (38).

(E4). $\quad \phi_{t}(n)=\phi_{t}^{0} \mathbb{I}_{\left\{n \in \overline{\mathcal{M}}_{t}^{0}\right\}}(n)+\phi_{t}^{1} \mathbb{I}_{\left\{n \in \overline{\mathcal{M}}_{t}^{1}\right\}}(n)$, with $\left\{\left(\phi_{t}^{s}\right)_{s=0,1}\right\}_{t=0}^{\infty}=\left\{\left(z_{t}^{s} / \bar{M}_{t}^{s}\right)_{s=0,1}\right\}_{t=0}^{\infty}$, where $\left\{\left(z_{t}^{s}\right)_{s=0,1}\right\}_{t=0}^{\infty}$ satisfies $(35)-(36)$, and $z_{t}=z_{t}^{0}+z_{t}^{1}$. 
(E5). $\quad \bar{M}_{t+1}^{0}$ is given by (33) with $\bar{M}_{0}^{0}=\theta_{0} M_{0}, \bar{M}_{t+1}^{1}$ is given by $(34)$ with $\bar{M}_{0}^{1}=\left(1-\theta_{0}\right) M_{0}+$ $B_{0}$, and (39) is satisfied. (The first two conditions imply that $\left\{\bar{M}_{t+1}\right\}_{t=0}^{\infty}$ follows (1) with $\bar{M}_{0}=M_{0}+B_{0}$ given, and $M_{t+1}=\bar{M}_{t}-q_{t} B_{t+1}$ for all $t \geq 0$.)

(E6). $\quad \overline{\mathfrak{m}}_{i t} \in \overline{\mathfrak{M}}_{t}, \bar{m}_{i t}=\bar{M}_{t}$ for a.e. $i \in \mathcal{I}$, and $\int_{\mathcal{I}} \int_{\mathcal{M}} \overline{\mathfrak{m}}_{i t}(d s) \mu(d i)=\mu(\mathcal{M})$ for all $\mathcal{M} \in$ $\mathcal{F}\left(\overline{\mathcal{M}}_{t}\right)$.

(E7). $\quad \mathfrak{b}_{i t+1} \in \mathfrak{B}_{t+1}, b_{i t+1}=B_{t+1}$ for a.e. $i \in \mathcal{I}$, and $\int_{\mathcal{I}} \int_{\mathcal{B}} \mathfrak{b}_{i t+1}(d s) \mu(d i)=\mu(\mathcal{B})$ for all $\mathcal{B} \in \mathcal{F}\left(\mathcal{B}_{t+1}\right)$.

(E8). $\quad \mathfrak{m}_{i t+1} \in \overline{\mathfrak{M}}_{t}$, and $m_{i t+1}=M_{t+1}$ for a.e. $i \in \mathcal{I}$.

(E9). For a.e. $i \in \mathcal{I}, \mathfrak{n}_{i t+1}^{-} \in \overline{\mathfrak{M}}_{t}, n_{i t+1}^{s-} / q_{t}=b_{i t+1}^{s}$ satisfies (28), $n_{i t+1}^{-}=q_{t} B_{t+1}$, and $\mathfrak{n}_{i t+1}^{-}(\mathcal{M})+\mathfrak{m}_{i t+1}(\mathcal{M})=\overline{\mathfrak{m}}_{i t}(\mathcal{M})$ for all $\mathcal{M} \in \mathcal{F}\left(\overline{\mathcal{M}}_{t}\right)$.

PART 2. Let agent's beliefs be as described in Property 1. Let $\theta_{0} \in(0,1)$ be the arbitrary number introduced in Part 1, and let

$$
\rho \in\left(\frac{1}{1+\Delta_{0}}, 1\right)
$$

be an arbitrary number, where $\Delta_{0} \equiv \frac{1-\theta_{0}}{\theta_{0}} \min \left(\frac{1-\theta_{0}}{\sup \mathbb{X}}, 1\right)$. Next, I construct a monetary equilibrium indexed by $\theta_{0}$. For all $t$, and a.e. $i, j \in \mathcal{I}$, set

$$
c_{i j t}=z \mathbb{I}_{\left\{i \in \mathcal{I}_{t}^{b}\right\}}(i), y_{i t}=z \mathbb{I}_{\left\{i \in \mathcal{I}_{t}^{s}\right\}}(i) \text {, and } h_{i t}=z \mathbb{I}_{\left\{i \in \mathcal{I}_{t}^{b}\right\}}(i)
$$

where $z$ is the unique solution to

$$
\beta \mathcal{L}^{\prime}(z)=1
$$

For all $t$, let

$$
\begin{aligned}
\bar{M}_{t}^{0} & =\theta_{0} M_{0} \\
\bar{M}_{t+1}^{1} & =\max \left(x_{t} \bar{M}_{t}, \bar{M}_{t}^{1}\right),
\end{aligned}
$$

where $\bar{M}_{t} \equiv \bar{M}_{t}^{0}+\bar{M}_{t}^{1}$, with

$$
\bar{M}_{0}^{1}=\left(1-\theta_{0}\right) M_{0}+B_{0},
$$

and $\bar{M}_{0}=M_{0}+B_{0}$ given by the initial conditions $M_{0}$ and $B_{0}$. For all $t \geq 0$, let

$$
M_{t}^{0}=\bar{M}_{0}^{0} \quad \text { and } \quad M_{t+1}^{1}=\max \left(1-\theta_{t}-x_{t}, 0\right) \bar{M}_{t},
$$


with $M_{0}^{1}=\left(1-\theta_{0}\right) M_{0}$ and $\theta_{t} \equiv \bar{M}_{t}^{0} / \bar{M}_{t}$. Then for all $t$, set

$$
\phi_{t}(n)= \begin{cases}\phi_{0}^{0} & \text { for } n \in\left[0, \bar{M}_{0}^{0}\right] \\ \phi_{t}^{1} & \text { for } n \in\left(\bar{M}_{0}^{0}, \bar{M}_{t}\right]\end{cases}
$$

with

$$
\phi_{0}^{0}=\frac{\rho z}{\bar{M}_{0}^{0}} \quad \text { and } \quad \phi_{t}^{1}=\frac{(1-\rho) z}{\bar{M}_{t}^{1}} .
$$

For every $t$ and all $s \in \mathcal{B}_{t+1}$, set

$$
q_{t}(s)=\min \left(\frac{1-\theta_{t}}{x_{t}}, 1\right) \equiv q_{t}
$$

Posit that each agent purchases $B_{t+1}^{s}$ bonds with notes of type $s$ in period $t \geq 0$, as follows

$$
\begin{aligned}
& B_{t+1}^{0}=0 \\
& B_{t+1}^{1}=x_{t} \bar{M}_{t} .
\end{aligned}
$$

For all $i \in \mathcal{I}$, and $t \geq 0$, let individual portfolios be

$$
\begin{aligned}
\overline{\mathfrak{m}}_{i t}(\mathcal{M}) & =\mathbb{I}_{\left\{i \bar{M}_{0}^{0} \in \mathcal{M}\right\}}(i) \bar{M}_{0}^{0}+\mathbb{I}_{\left\{\bar{M}_{0}^{0}+i M_{t+1}^{1} \in \mathcal{M}\right\}}(i) M_{t+1}^{1} \\
& +\mathbb{I}_{\left\{\bar{M}_{0}^{0}+M_{t+1}^{1}+i q_{t} B_{t+1} \in \mathcal{M}\right\}}(i) q_{t} B_{t+1} \\
\mathfrak{m}_{i t+1}(\mathcal{M}) & =\left\{\mathbb{I}_{\left\{i M_{0}^{0} \in \mathcal{M}\right\}}(i) M_{0}^{0}+\mathbb{I}_{\left\{M_{0}^{0}+i M_{t+1}^{1} \in \mathcal{M}\right\}}(i) M_{t+1}^{1}\right\} \mathbb{I}_{\left\{\mathcal{M} \in \mathcal{F}\left(\mathcal{M}_{t+1}\right)\right\}} \\
\mathfrak{n}_{i t+1}^{-}(\mathcal{M}) & =\overline{\mathfrak{m}}_{i t}(\mathcal{M})-\mathfrak{m}_{i t+1}(\mathcal{M})
\end{aligned}
$$

for all $\mathcal{M} \in \mathcal{F}\left(\overline{\mathcal{M}}_{t}\right)$, and for all $\mathcal{B} \in \mathcal{F}\left(\mathcal{B}_{t+1}\right)$,

$$
\mathfrak{b}_{i t+1}(\mathcal{B})=\mathbb{I}_{\left\{i B_{t+1} \in \mathcal{B}\right\}}(i) B_{t+1}
$$

The proposed paths (47) and (48) satisfy Properties 2 and 3. From (42) and (48), $\phi_{t} \overline{\boldsymbol{M}}_{t}=$ $z \in\left(0, c^{*}\right)$, so the $c_{i j t}, y_{i t}$, and $h_{i t}$ proposed in (41) satisfy (E1) and (E2).

With (48), $\bar{q}_{t}^{0} \equiv \phi_{t+1}^{1} / \phi_{t+1}^{0}$ becomes

$$
\bar{q}_{t}^{0}=\frac{1-\rho}{\rho} \frac{\bar{M}_{0}^{0}}{\bar{M}_{t+1}^{1}} .
$$

Since (44) and (49) imply

$$
\bar{M}_{t+1}^{1}=\max \left(\frac{1}{q_{t}}, 1\right) \bar{M}_{t}^{1},
$$

I have

$$
\bar{q}_{t+1}^{0}=\min \left(q_{t+1}, 1\right) \bar{q}_{t}^{0} \leq \bar{q}_{t}^{0} \quad \text { for all } t
$$


Notice that

$$
\begin{aligned}
\bar{q}_{0}^{0} & =\frac{1-\rho}{\rho} \frac{\theta_{0}}{1-\theta_{0}} \min \left(q_{0}, 1\right) \leq \frac{1-\rho}{\rho} \frac{\theta_{0}}{1-\theta_{0}} \\
& <\min \left(\frac{1-\theta_{0}}{\sup \mathbb{X}}, 1\right) \leq \min \left(\frac{1-\theta_{t}}{x_{t}}, 1\right)=q_{t},
\end{aligned}
$$

where the strict inequality follows from (40), and the last inequality uses the fact that $\theta_{t+1} \leq \theta_{t}$ for all $t$. Therefore, combining (58) and (59),

$$
\bar{q}_{t}^{0} \leq \bar{q}_{0}^{0}<q_{t} \leq 1=\bar{q}_{t}^{1} \quad \text { for all } t
$$

Given (56) and (60), (38) reduces to

$$
q_{t}= \begin{cases}1 & \text { if } x_{t} \leq 1-\theta_{t} \\ \frac{1-\theta_{t}}{x_{t}} & \text { if } 1-\theta_{t}<x_{t}<\frac{1-\theta_{t}}{\bar{q}_{t}^{0}} \\ \bar{q}_{t}^{0} & \text { if } \frac{1-\theta_{t}}{\bar{q}_{t}^{0}} \leq x_{t} \leq \frac{1}{\bar{q}_{t}^{0}} \\ \frac{1}{x_{t}} & \text { if } \frac{1}{\bar{q}_{t}^{0}}<x_{t} .\end{cases}
$$

For all $t,(58),(59)$ and $\theta_{t+1} \leq \theta_{t}$ imply

$$
\bar{q}_{t}^{0} \leq \bar{q}_{0}^{0} \leq \frac{1-\rho}{\rho} \frac{\theta_{0}}{1-\theta_{0}}<\min \left(\frac{1-\theta_{0}}{\sup \mathbb{X}}, 1\right) \leq \frac{1-\theta_{0}}{\sup \mathbb{X}} \leq \frac{1-\theta_{t}}{x_{t}},
$$

where the strict inequality follows from (40). Thus (61) (and therefore (38)) reduces to (49), so (E3) is satisfied.

With (50), notice that for all $t,(33)$ and (34) reduce to (43) and (57), respectively, with initial conditions $\bar{M}_{0}^{0}=\theta_{0} M_{0}$ and $\bar{M}_{0}^{1}=\left(1-\theta_{0}\right) M_{0}+B_{0}$ given by (43) and (45). Moreover, (43) and (57) imply

$$
\bar{M}_{t+1}=\max \left(x_{t}+\theta_{t}, 1\right) \bar{M}_{t}
$$

which is the same as (1) with $q_{t}$ given by (49). Also, with (49), the conditions in (39) reduce to the conditions in (46). Hence (E5) is satisfied.

As defined in (52), $\overline{\mathfrak{m}}_{i t}$ satisfies $\overline{\mathfrak{m}}_{i t} \in \overline{\mathfrak{M}}_{t}$, and $\bar{m}_{i t} \equiv \overline{\mathfrak{m}}_{i t}\left(\overline{\mathcal{M}}_{t}\right)=\bar{M}_{0}^{0}+M_{t+1}^{1}+q_{t} B_{t+1}=\bar{M}_{t}$ 
(the last equality follows from (43), (46), (49), (50), and (51)). Also, for any $\mathcal{M} \in \mathcal{F}\left(\overline{\mathcal{M}}_{t}\right)$,

$$
\begin{aligned}
\int_{\mathcal{I}} \int_{\mathcal{M}} \overline{\mathfrak{m}}_{i t}(d s) \mu(d i) & =\int_{\mathcal{I}} \overline{\mathfrak{m}}_{i t}(\mathcal{M}) \mu(d i) \\
& =\int_{\mathcal{I}} \mathbb{I}_{\left\{i \bar{M}_{0}^{0} \in \mathcal{M}\right\}}(i) \bar{M}_{0}^{0} \mu(d i)+\int_{\mathcal{I}} \mathbb{I}_{\left\{\bar{M}_{0}^{0}+i M_{t+1}^{1} \in \mathcal{M}\right\}}(i) M_{t+1}^{1} \mu(d i) \\
& +\int_{\mathcal{I}} \mathbb{I}_{\left\{\bar{M}_{0}^{0}+M_{t+1}^{1}+i q_{t} B_{t+1} \in \mathcal{M}\right\}}(i) q_{t} B_{t+1} \mu(d i) \\
& =\mu\left(\left\{i \bar{M}_{0}^{0} \in \mathcal{M}\right\}\right) \bar{M}_{0}^{0}+\mu\left(\left\{\bar{M}_{0}^{0}+i M_{t+1}^{1} \in \mathcal{M}\right\}\right) M_{t+1}^{1} \\
& +\mu\left(\left\{\bar{M}_{0}^{0}+M_{t+1}^{1}+i q_{t} B_{t+1} \in \mathcal{M}\right\}\right) q_{t} B_{t+1} \\
& =\mu(\mathcal{M}),
\end{aligned}
$$

where the last equality follows from the relative dilation invariance of the Lebesgue measure (Theorem D in Halmos, 1974, p. 64). Hence Property 4 and (E6) are satisfied.

As defined in $(55), \mathfrak{b}_{i t+1} \in \mathfrak{B}_{t+1}$, and $b_{i t+1} \equiv \mathfrak{b}_{i t+1}\left(\mathcal{B}_{t+1}\right)=\mathbb{I}_{\left\{i B_{t+1} \in \mathcal{B}_{t+1}\right\}}(i) B_{t+1}=B_{t+1}$. Also, for all $\mathcal{B} \in \mathcal{F}\left(\mathcal{B}_{t+1}\right)$,

$$
\begin{aligned}
\int_{\mathcal{I}} \int_{\mathcal{B}} \mathfrak{b}_{i t+1}(d s) \mu(d i) & =\int_{\mathcal{I}} \mathfrak{b}_{i t+1}(\mathcal{B}) \mu(d i) \\
& =\int_{\mathcal{I}} \mathbb{I}_{\left\{i B_{t+1} \in \mathcal{B}\right\}}(i) B_{t+1} \mu(d i) \\
& =\mu\left(\left\{i B_{t+1} \in \mathcal{B}\right\}\right) B_{t+1} \\
& =\mu(\mathcal{B}) .
\end{aligned}
$$

Hence (E7) is satisfied. As defined in (53), $\mathfrak{m}_{i t+1}$ satisfies $\mathfrak{m}_{i t+1} \in \overline{\mathfrak{M}}_{t}$, and $\mathfrak{m}_{i t+1}\left(\mathcal{M}_{t+1}\right)=$ $M_{0}^{0}+M_{t+1}^{1}=M_{t+1}$ (since by (46), $M_{t+1}^{0}=M_{0}^{0}$ for all $t$ ) so (E8) is satisfied.

From (60), $\bar{q}_{t}^{0}<q_{t}$ for all $t$, so (28) for $s=0$ reduces to (50). Also, from (49), $q_{t} \leq 1=\bar{q}_{t}^{1}$ for all $t$, so $(28)$ for $s=1$ reduces to

$$
B_{t+1}^{1} \begin{cases}\in\left[0, \bar{M}_{t}^{1}\right] & \text { if } q_{t}=1 \\ =\frac{\bar{M}_{t}^{1}}{q_{t}} & \text { if } q_{t}<1 .\end{cases}
$$

With (49), it is clear that (51) satisfies (62), so I can conclude that (50) and (51) satisfy (28). As defined in (54), $\mathfrak{n}_{i t+1}^{-} \in \overline{\mathfrak{M}}_{t}$, and evaluating (54) at $\mathcal{M}=\overline{\mathcal{M}}_{t}^{s}$ implies $n_{i t+1}^{s-}=\bar{M}_{t}^{s}-M_{t+1}^{s}=$ $q_{t} B_{t+1}^{s}$, where the last equality follows from (43), (46), (49), (50), and (51). Similarly, evaluating (54) at $\mathcal{M}=\overline{\mathcal{M}}_{t}$ implies $n_{i t+1}^{-}=\bar{M}_{t}-M_{t+1}=q_{t} B_{t+1}$. Hence (E9) is satisfied.

With (49), (50) and (60), the Euler equations (35) and (36) reduce to

$$
z_{t}^{s}=\beta E_{t}\left[\mathcal{L}^{\prime}\left(z_{t+1}\right) z_{t+1}^{s}\right] \quad \text { for } s=0,1,
$$


which combined, and using $z_{t}^{0}+z_{t}^{1}=z_{t}$, imply

$$
z_{t}=\beta E_{t}\left[\mathcal{L}^{\prime}\left(z_{t+1}\right) z_{t+1}\right]
$$

But then notice that the constant path $\left\{z_{t}^{0}, z_{t}^{1}, z_{t}\right\}_{t=0}^{\infty}=\{\rho z,(1-\rho) z, z\}_{t=0}^{\infty}$, with $z$ defined by (42), satisfies (63) and (64). Thus, (47) and (48) satisfy (E4).

In summary, for a given $\theta_{0}$, the proposed allocations and prices constitute a monetary equilibrium. Since $\theta_{0}$ is arbitrary, the construction characterizes a continuum of monetary equilibria, one for each $\theta_{0} \in(0,1)$.

PART 3. In the equilibrium described in Part 2, $B_{t+1}=x_{t} \bar{M}_{t}>0$, and bonds are always held. The price of bonds satisfies $0<q_{t} \leq 1$ for all $t$ (with $q_{t}<1$ if $1-\theta_{t}<x_{t}$ ), and

$$
i_{t+1}=\max \left(\frac{x_{t}}{1-\theta_{t}}-1,0\right) .
$$

The real value of the money holdings brought into the decentralized market of period $t+1$ is

$$
\int_{\mathcal{I}}\left(\phi_{t+1} \cdot \mathfrak{m}_{i t+1}\right) \mu(d i)=\rho z+\max \left(1-\frac{x_{t}}{1-\theta_{t}}, 0\right)(1-\rho) z>0 .
$$

Hence $\int_{\mathcal{I}}\left(\phi_{t+1} \cdot \mathfrak{m}_{i t+1}\right) \mu(d i)>0$ for all $t$, even if $i_{t}>0$ as is the case whenever the realization of the relative size of the open-market operation, $x_{t}$, lies in the interval $\left(1-\theta_{t}, \sup \mathbb{X}\right]$.

Proof of Proposition 3. Let the notation be as in the proof of Proposition 2. Also as in that proof, let the sequence of partitions $\left\{\left\langle\overline{\mathcal{M}}_{t}^{0}, \overline{\mathcal{M}}_{t}^{1}\right\rangle\right\}_{t=0}^{\infty}$ be given by (22)-(24), and $M_{1}^{s}=\bar{M}_{0}^{s}-q_{0} B_{1}^{s}$ for $s=0,1, \bar{M}_{1}^{0}=M_{1}^{0}$, and $\bar{M}_{1}^{1}=M_{1}^{1}+B_{1}$. In addition, the current assumptions on $\left\{B_{t}\right\}_{t=0}^{\infty}$ imply $M_{0}^{0}=\bar{M}_{0}^{0}=\theta_{0} M_{0}, M_{0}^{1}=\bar{M}_{0}^{1}=\left(1-\theta_{0}\right) M_{0}$, and $\bar{M}_{t}^{s}=M_{t}^{s}=\bar{M}_{1}^{s}$ for all $t \geq 2$. To illustrate the possibility of negative nominal rates, I construct an equilibrium as follows. Begin with the optimization problem of an agent in the centralized market of period $t \geq 1$,

$$
\max _{0 \leq \overline{\boldsymbol{m}}_{t+1}}\left[-\boldsymbol{\phi}_{t} \overline{\boldsymbol{m}}_{t+1}+\beta \mathcal{L}\left(\boldsymbol{\phi}_{t+1} \overline{\boldsymbol{m}}_{t+1}\right)\right] .
$$

This is the special case of (26) that obtains by replacing the function $\lambda_{t+1}\left(\overline{\boldsymbol{m}}_{t+1}, \boldsymbol{b}_{t+1}\right)$ with $\phi_{t+1} \overline{\boldsymbol{m}}_{t+1}$. (Since $B_{t+1}=0$ for $t \geq 1$, this is a purely monetary economy from the centralized market of date 1 onwards.) The Euler equation for money of type $s$ is

$$
-\phi_{t}^{s}+\beta \mathcal{L}^{\prime}\left(\phi_{t+1} \overline{\boldsymbol{m}}_{t+1}\right) \phi_{t+1}^{s} \leq 0, \quad \text { " }=\text { " if } \bar{m}_{t+1}^{s}>0, \quad \text { for all } t \geq 1
$$


Focus on an equilibrium with $\phi_{t}(n)=\sum_{s=0}^{1} \phi_{t}^{s} \mathbb{I}_{\left\{n \in \overline{\mathcal{M}}_{t}^{s}\right\}}(n)$, where $\phi_{t}^{0}=0$ and $\phi_{t}^{1}=\phi_{1}^{1}>0$, for all $t \geq 1$. Since $\bar{M}_{t}^{1}=\bar{M}_{1}^{1}$ for all $t \geq 1$, real balances are constant from period 1 onwards in the equilibrium under consideration, i.e., $\phi_{t}^{1} \bar{M}_{t}^{1}=\phi_{1}^{1} \bar{M}_{1}^{1} \equiv z$ for all $t \geq 1$, where $z$ is the unique solution to (42). Hence given $z$ (which is guaranteed to be strictly positive),

$$
\phi_{t}^{1}=\phi_{1}^{1}=\frac{z}{\bar{M}_{1}^{1}} \quad \text { for all } t \geq 1
$$

At the end of period 0 , an agent who enters the bond market with $\overline{\boldsymbol{m}}_{0}=\left(\bar{m}_{0}^{0}, \bar{m}_{0}^{1}\right)$ solves

$$
\max _{0 \leq q_{0} \boldsymbol{b}_{1} \leq \overline{\boldsymbol{m}}_{0}} \beta \mathcal{L}\left[\bar{\lambda}\left(\overline{\boldsymbol{m}}_{0}, \boldsymbol{b}_{1}\right)\right]
$$

where $\bar{\lambda}\left(\overline{\boldsymbol{m}}_{0}, \boldsymbol{b}_{1}\right) \equiv \phi_{1}^{1} \bar{m}_{0}^{1}+\phi_{1}^{1} b_{1}^{0}+\left(1-q_{0}\right) \phi_{1}^{1} b_{1}^{1}$. This is just a special case of the inner maximization in (26), for $t=0$, with $\phi_{1}^{0}=0$, and no uncertainty. The implied individual bond demands are $b_{1}^{0}=\bar{m}_{0}^{0} / q_{0}$, and $b_{1}^{1}=g_{0}^{1}\left(\bar{m}_{0}^{1}\right)$, where $g_{0}^{1}(\cdot)$ is as in (28). In the centralized market of period 0 , the agent solves

$$
\max _{0 \leq \overline{\boldsymbol{m}}_{0}}\left\{-\phi_{0} \overline{\boldsymbol{m}}_{0}+\beta \mathcal{L}\left[\bar{\lambda}^{*}\left(\overline{\boldsymbol{m}}_{0}\right)\right]\right\}
$$

where $\bar{\lambda}^{*}\left(\overline{\boldsymbol{m}}_{0}\right) \equiv \bar{\lambda}\left(\overline{\boldsymbol{m}}_{0}, \bar{m}_{0}^{0} / q_{0}, g_{0}^{1}\left(\bar{m}_{0}^{1}\right)\right)=\phi_{1}^{1} \bar{m}_{0}^{0} / q_{0}+\max \left(1 / q_{0}, 1\right) \phi_{1}^{1} \bar{m}_{0}^{1}$. Focus on an equilibrium in which both types of moneys are held at $t=0$. Then the Euler equations corresponding to this problem are

$$
\phi_{0}^{0}=\beta \mathcal{L}^{\prime}\left[\bar{\lambda}^{*}\left(\overline{\boldsymbol{m}}_{0}\right)\right] \frac{1}{q_{0}} \phi_{1}^{1} \quad \text { and } \quad \phi_{0}^{1}=\beta \mathcal{L}^{\prime}\left[\bar{\lambda}^{*}\left(\overline{\boldsymbol{m}}_{0}\right)\right] \max \left(\frac{1}{q_{0}}, 1\right) \phi_{1}^{1} .
$$

In equilibrium, $\overline{\boldsymbol{m}}_{0}=\overline{\boldsymbol{M}}_{0}$ and $\bar{\lambda}^{*}\left(\overline{\boldsymbol{M}}_{0}\right)=z$, so these conditions become

$$
\begin{aligned}
\phi_{0}^{0} & =\frac{1}{q_{0}} \phi_{1}^{1} \\
\phi_{0}^{1} & =\max \left(\frac{1}{q_{0}}, 1\right) \phi_{1}^{1} .
\end{aligned}
$$

Let $G_{0}\left(\overline{\boldsymbol{M}}_{0}\right) \equiv \bar{M}_{0}^{0} / q_{0}+g_{0}^{1}\left(\bar{M}_{0}^{1}\right)$ denote the aggregate demand for bonds at $t=0$. The bond-market equilibrium condition at $t=0, G_{0}\left(\overline{\boldsymbol{M}}_{0}\right)=B_{1}$, yields

$$
q_{0}= \begin{cases}\frac{\theta_{0}}{x_{0}} & \text { if } x_{0}<\theta_{0} \\ 1 & \text { if } \theta_{0} \leq x_{0} \leq 1 \\ \frac{1}{x_{0}} & \text { if } 1<x_{0}\end{cases}
$$

The individual bond demands, the constraint $q_{0} B_{1}^{s}+M_{1}^{s}=\bar{M}_{0}^{s}$, and (68) imply

$$
M_{1}^{0}=0 \quad \text { and } \quad q_{0} B_{1}^{0}=\theta_{0} M_{0}
$$


and

$$
M_{1}^{1}=\left\{\begin{array}{ll}
\left(1-\theta_{0}\right) M_{0} & \text { if } x_{0}<\theta_{0} \\
\left(1-x_{0}\right) M_{0} & \text { if } \theta_{0} \leq x_{0} \leq 1 \\
0 & \text { if } 1<x_{0}
\end{array} \quad \text { and } \quad q_{0} B_{1}^{1}= \begin{cases}0 & \text { if } x_{0}<\theta_{0} \\
\left(x_{0}-\theta_{0}\right) M_{0} & \text { if } \theta_{0} \leq x_{0} \leq 1 \\
\left(1-\theta_{0}\right) M_{0} & \text { if } 1<x_{0} .\end{cases}\right.
$$

Since $\bar{M}_{1}^{1}=\bar{M}_{0}^{1}-q_{0} B_{1}^{1}+B_{1}=\left(1-\theta_{0}+x_{0}\right) M_{0}-q_{0} B_{1}^{1},(70)$ implies

$$
\bar{M}_{1}^{1}= \begin{cases}\left(1-\theta_{0}+x_{0}\right) M_{0} & \text { if } x_{0}<\theta_{0} \\ M_{0} & \text { if } \theta_{0} \leq x_{0} \leq 1 \\ x_{0} M_{0} & \text { if } 1<x_{0}\end{cases}
$$

Combined, (65) and (71) imply

$$
\phi_{1}^{1}= \begin{cases}\frac{z}{\left(1-\theta_{0}+x_{0}\right) M_{0}} & \text { if } x_{0}<\theta_{0} \\ \frac{z}{M_{0}} & \text { if } \theta_{0} \leq x_{0} \leq 1 \\ \frac{z}{x_{0} M_{0}} & \text { if } 1<x_{0}\end{cases}
$$

Finally, with (68) and (72), (66) and (67) become

$$
\phi_{0}^{0}=\left\{\begin{array}{ll}
\frac{x_{0} z}{\theta_{0}\left(1-\theta_{0}+x_{0}\right) M_{0}} & \text { if } x_{0}<\theta_{0} \\
\frac{z}{M_{0}} & \text { if } \theta_{0} \leq x_{0}
\end{array} \quad \text { and } \quad \phi_{0}^{1}= \begin{cases}\frac{z}{\left(1-\theta_{0}+x_{0}\right) M_{0}} & \text { if } x_{0}<\theta_{0} \\
\frac{z}{M_{0}} & \text { if } \theta_{0} \leq x_{0} .\end{cases}\right.
$$

At this point a family of monetary equilibria with moneyspots, indexed by the arbitrary number $\theta_{0} \in(0,1)$ has been constructed. Given a value for $\theta_{0}$, the equilibrium can be summarized as follows. The prices of bonds and the two types of money are $q_{0}$ and $\left\{\left(\phi_{t}^{s}\right)_{s=0,1}\right\}_{t=0}^{\infty}$, where $q_{0}$ is given by (68), $\phi_{0}^{0}$ and $\phi_{0}^{1}$ are given by $(73), \phi_{t}^{0}=0$ for $t \geq 1$, and $\phi_{t}^{1}=\phi_{1}^{1}$ for $t \geq 1$, with $\phi_{1}^{1}$ given by (72). Each agent carries $M_{1}^{s}$ units of money of type $s$ into period 1 and uses $q_{0} B_{1}^{s}$ units of money of type $s$ to purchase bonds in period 0 , with the corresponding expressions given by (69) and (70), for $s=0$ and $s=1$, respectively. Along the equilibrium path, $M_{0}^{0}=\bar{M}_{0}^{0}=\theta_{0} M_{0}, M_{0}^{1}=\bar{M}_{0}^{1}=\left(1-\theta_{0}\right) M_{0}, M_{1}^{0}=\bar{M}_{1}^{0}=0, M_{1}^{1}$ is as in $(70), \bar{M}_{1}^{1}$ as in (71), and $\left\{\left(M_{t}^{s}\right)_{s=0,1}\right\}_{t=2}^{\infty}=\left\{\left(\bar{M}_{t}^{s}\right)_{s=0,1}\right\}_{t=2}^{\infty}=\left\{\left(\bar{M}_{1}^{s}\right)_{s=0,1}\right\}_{t=2}^{\infty}$.

The desired result is immediate from (68): the equilibrium nominal interest rate on government bonds between period 0 and period 1, i.e., $i_{1}=1 / q_{0}-1$, is

$$
i_{1}= \begin{cases}\frac{x_{0}}{\theta_{0}}-1 & \text { if } x_{0}<\theta_{0} \\ 0 & \text { if } \theta_{0} \leq x_{0} \leq 1 \\ x_{0}-1 & \text { if } 1<x_{0}\end{cases}
$$

which is negative if the size of the open-market operation is small enough, i.e., $x_{0}<\theta_{0}$. Equivalently, for any $x_{0}<1$, there exists a continuum of moneyspot equilibria with $i_{1}<0$, indexed by the arbitrary number $\theta_{0} \in\left(x_{0}, 1\right)$. 


\section{B Subsidiary proofs}

The following lemma provides closed-form expressions for the value functions that will summarize the maximum expected utility attainable by an agent $i \in \mathcal{I}$ in any equilibrium.

Lemma 1 Agent $i$ 's values are:

$$
\begin{aligned}
W_{t}\left(\mathfrak{a}_{i t}\right) & =\phi_{t} \cdot \mathfrak{m}_{i t}+\phi_{t} \cdot\left(\mathfrak{b}_{i t} \omega^{-1}\right)+K_{t} \\
V_{t}\left(\mathfrak{a}_{i t}\right) & =\mathcal{L}\left[\Lambda_{t}\left(\mathfrak{a}_{i t}\right)\right]+K_{t} \\
U_{t}\left(\overline{\mathfrak{m}}_{i t}\right) & =\max _{\mathfrak{a}_{i t+1}, \mathfrak{n}_{i t+1}^{-}} \beta E_{t}\left\{\mathcal{L}\left[\Lambda_{t+1}\left(\mathfrak{a}_{i t+1}\right)\right]+K_{t+1}\right\},
\end{aligned}
$$

where $\left\{K_{t}\right\}$ satisfies

$$
K_{t}=\Delta_{t}+\beta E_{t} K_{t+1},
$$

$\Delta_{t}$ is given by

$$
\begin{gathered}
\Delta_{t} \equiv \max _{\overline{\mathfrak{m}}_{i t} \in \overline{\mathfrak{M}}_{t}}\left\{-\phi_{t} \cdot \overline{\mathfrak{m}}_{i t}+\max _{\mathfrak{a}_{i t+1}, \mathfrak{n}_{i t+1}^{-}} \beta E_{t} \mathcal{L}\left[\Lambda_{t+1}\left(\mathfrak{a}_{i t+1}\right)\right]\right\} \\
\text { s.t. } \mathfrak{n}_{i t+1}^{-}(\mathcal{M})+\mathfrak{m}_{i t+1}(\mathcal{M})=\overline{\mathfrak{m}}_{i t}(\mathcal{M}) \text { for all } \mathcal{M} \in \mathcal{F}\left(\overline{\mathcal{M}}_{t}\right), \\
q_{t} \cdot \mathfrak{b}_{i t+1}=\mathfrak{n}_{i t+1}^{-}\left(\overline{\mathcal{M}}_{t}\right), \mathfrak{m}_{i t+1}, \mathfrak{n}_{i t+1}^{-} \in \overline{\mathfrak{M}}_{t}, \mathfrak{b}_{i t+1} \in \mathfrak{B}_{t+1},
\end{gathered}
$$

and the maximization on the right side of (76) is subject to (79) and (80).

Proof of Lemma 1. Agent $i$ 's value in the centralized market is given by (10). Substitute (11) into the objective function on the right side of (10) to get (74), where

$$
K_{t}=\max _{\overline{\mathfrak{m}}_{i t} \in \overline{\mathfrak{M}}_{t}}\left\{-\phi_{t} \cdot \overline{\mathfrak{m}}_{i t}+U_{t}\left(\overline{\mathfrak{m}}_{i t}\right)\right\} .
$$

Let $\left\langle c_{t}\left(\mathfrak{a}_{i t}, \mathfrak{a}_{j t}\right),\left(\mathfrak{m}_{t}^{\prime}\left(\mathfrak{a}_{i t}, \mathfrak{a}_{j t}\right), \mathfrak{b}_{t}^{\prime}\left(\mathfrak{a}_{i t}, \mathfrak{a}_{j t}\right)\right)\right\rangle$ denote the bargaining outcome in a bilateral trade between agent $i$ who is acting as buyer and holding portfolio $\mathfrak{a}_{i t}=\left(\mathfrak{m}_{i t}, \mathfrak{b}_{i t}\right)$, and agent $j$ who is acting as seller and holding portfolio $\mathfrak{a}_{j t}=\left(\mathfrak{m}_{j t}, \mathfrak{b}_{j t}\right)$, at time $t$. Upon entering the decentralized market of period $t$ holding $\mathfrak{a}_{i t}=\left(\mathfrak{m}_{i t}, \mathfrak{b}_{i t}\right)$, with $\mathfrak{m}_{i t} \in \overline{\mathfrak{M}}_{t-1}$ and $\mathfrak{b}_{i t} \in \mathfrak{B}_{t}$, agent $i$ 's value is

$$
\begin{aligned}
V_{t}\left(\mathfrak{a}_{i t}\right) & =\alpha \int\left\{u\left[c_{t}\left(\mathfrak{a}_{i t}, \mathfrak{a}_{j t}\right)\right]+E_{t} W_{t}\left[\mathfrak{m}_{i t} \ominus \mathfrak{m}_{t}^{\prime}\left(\mathfrak{a}_{i t}, \mathfrak{a}_{j t}\right), \mathfrak{b}_{i t} \ominus \mathfrak{b}_{t}^{\prime}\left(\mathfrak{a}_{i t}, \mathfrak{a}_{j t}\right)\right]\right\} \digamma\left(d \mathfrak{a}_{j t}\right) \\
& +\alpha \int\left\{-c_{t}\left(\mathfrak{a}_{j t}, \mathfrak{a}_{i t}\right)+E_{t} W_{t}\left[\mathfrak{m}_{i t} \oplus \mathfrak{m}_{t}^{\prime}\left(\mathfrak{a}_{j t}, \mathfrak{a}_{i t}\right), \mathfrak{b}_{i t} \oplus \mathfrak{b}_{t}^{\prime}\left(\mathfrak{a}_{j t}, \mathfrak{a}_{i t}\right)\right]\right\} \digamma\left(d \mathfrak{a}_{j t}\right) \\
& +(1-\alpha) E_{t} W_{t}\left(\mathfrak{m}_{i t}, \mathfrak{b}_{i t}\right)
\end{aligned}
$$


where the expectation operator $E_{t}$ is with respect to the measure $\nu_{t}$ over assignment realizations of the redemption lottery, and $\digamma$ is the probability measure over portfolios in the population. Use (74) and the fact that $E_{t} W_{t}\left(\mathfrak{m}_{i t} \oplus \mathfrak{m}_{t}^{\prime}, \mathfrak{b}_{i t} \oplus \mathfrak{b}_{t}^{\prime}\right)-E_{t} W_{t}\left(\mathfrak{m}_{i t}, \mathfrak{b}_{i t}\right)=E_{t} W_{t}\left(\mathfrak{m}_{i t}, \mathfrak{b}_{i t}\right)-$ $E_{t} W_{t}\left(\mathfrak{m}_{i t} \ominus \mathfrak{m}_{t}^{\prime}, \mathfrak{b}_{i t} \ominus \mathfrak{b}_{t}^{\prime}\right)=\Lambda_{t}\left(\mathfrak{m}_{t}^{\prime}, \mathfrak{b}_{t}^{\prime}\right)$, to arrive at

$$
\begin{aligned}
V_{t}\left(\mathfrak{a}_{i t}\right) & =\alpha \int\left\{u\left[c_{t}\left(\mathfrak{a}_{i t}, \mathfrak{a}_{j t}\right)\right]-\Lambda_{t}\left(\mathfrak{m}_{t}^{\prime}\left(\mathfrak{a}_{i t}, \mathfrak{a}_{j t}\right), \mathfrak{b}_{t}^{\prime}\left(\mathfrak{a}_{i t}, \mathfrak{a}_{j t}\right)\right)\right\} \digamma\left(d \mathfrak{a}_{j t}\right) \\
& +\alpha \int\left\{-c_{t}\left(\mathfrak{a}_{j t}, \mathfrak{a}_{i t}\right)+\Lambda_{t}\left(\mathfrak{m}_{t}^{\prime}\left(\mathfrak{a}_{j t}, \mathfrak{a}_{i t}\right), \mathfrak{b}_{t}^{\prime}\left(\mathfrak{a}_{j t}, \mathfrak{a}_{i t}\right)\right)\right\} \digamma\left(d \mathfrak{a}_{j t}\right) \\
& +\Lambda_{t}\left(\mathfrak{m}_{i t}, \mathfrak{b}_{i t}\right)+K_{t} .
\end{aligned}
$$

The bargaining outcome implies $\Lambda_{t}\left(\mathfrak{m}_{t}^{\prime}\left(\mathfrak{a}_{i t}, \mathfrak{a}_{j t}\right), \mathfrak{b}_{t}^{\prime}\left(\mathfrak{a}_{i t}, \mathfrak{a}_{j t}\right)\right)=c_{t}\left(\mathfrak{a}_{i t}, \mathfrak{a}_{j t}\right)=c\left(\Lambda_{t}\left(\mathfrak{m}_{i t}, \mathfrak{b}_{i t}\right)\right)$, so

$$
V_{t}\left(\mathfrak{a}_{i t}\right)=\alpha\left\{u\left[c\left(\Lambda_{t}\left(\mathfrak{m}_{i t}, \mathfrak{b}_{i t}\right)\right)\right]-c\left(\Lambda_{t}\left(\mathfrak{m}_{i t}, \mathfrak{b}_{i t}\right)\right)\right\}+\Lambda_{t}\left(\mathfrak{m}_{i t}, \mathfrak{b}_{i t}\right)+K_{t}
$$

which given the notation introduced in (5), is the same as (75). Substitute (75) into (6) to obtain (76). Finally, substitute (76) into (81) to arrive at (77), with $\Delta_{t}$ given by (78). The constraints (79) and (80) are the constraints to the maximization in (6).

According to Lemma $1, W_{t}\left(\mathfrak{a}_{t}\right)$ and $V_{t}\left(\mathfrak{a}_{t}\right)$ are separable functions of the agent's asset holdings, e.g., $W_{t}\left(\mathfrak{a}_{t}\right)$ is composed of the real value of the current post-redemption set of assets, $\phi_{t}$. $\mathfrak{m}_{i t}+\phi_{t} \cdot\left(\mathfrak{b}_{i t} \omega^{-1}\right)$, plus the continuation utility, $K_{t}$, which is independent of the current asset position. The term $\Delta_{t}$ in (78) is the "flow utility" associated with $K_{t}$, which summarizes the flow value of the agent's optimization problems in the competitive markets of period $t$.

The following lemma provides a sharper characterization of the solution to the optimization problems that an agent faces in the competitive markets of period $t$ in a monetary equilibrium that satisfies Properties 1 and 2 .

Lemma 2 Let $\lambda_{t+1}: \mathbb{R}^{4} \rightarrow \mathbb{R}$ be defined by $\lambda_{t+1}\left(\overline{\boldsymbol{m}}_{i t}, \boldsymbol{b}_{i t+1}\right)=\boldsymbol{\phi}_{t+1}\left(\overline{\boldsymbol{m}}_{i t}-q_{t} \boldsymbol{b}_{i t+1}\right)+\phi_{t+1}^{1} b_{i t+1}$. Suppose that $\left\{\left\langle\overline{\mathcal{M}}_{t+1}^{0}, \overline{\mathcal{M}}_{t+1}^{1}\right\rangle\right\}_{t=0}^{\infty}$ and $\left\{\phi_{t}\right\}_{t=0}^{\infty}$ satisfy Properties 1 and 2. A solution to agent $i$ 's time-t optimization problem, (13), is a collection of measures $\left(\overline{\mathfrak{m}}_{i t}, \mathfrak{m}_{i t+1}, \mathfrak{b}_{i t+1}, \mathfrak{n}_{i t+1}^{-}\right)$that satisfy $\overline{\mathfrak{m}}_{i t} \in \overline{\mathfrak{M}}_{t}$, (7) with equality, and (9), with associated quantities $\left(\bar{m}_{i t}^{s}, m_{i t+1}^{s}, b_{i t+1}^{s}, n_{i t+1}^{s-}\right)_{s=0,1}$ that achieve

$$
\Delta_{t}=\max _{\overline{\boldsymbol{m}}_{i t} \in \mathbb{R}^{+} \times \mathbb{R}^{+}}\left\{-\boldsymbol{\phi}_{t} \overline{\boldsymbol{m}}_{i t}+\max _{0 \leq q_{t} \boldsymbol{b}_{i t+1} \leq \overline{\boldsymbol{m}}_{i t}} \beta E_{t} \mathcal{L}\left[\lambda_{t+1}\left(\overline{\boldsymbol{m}}_{i t}, \boldsymbol{b}_{i t+1}\right)\right]\right\}
$$

with $m_{i t+1}^{s}=\bar{m}_{i t}^{s}-q_{t} b_{i t+1}^{s}$ and $n_{i t+1}^{s-}=q_{t} b_{i t+1}^{s}$ for $s=0,1$, and $b_{i t+1}=b_{i t+1}^{0}+b_{i t+1}^{1}$. 
Proof of Lemma 2. Given Property 2, $\phi_{t} \cdot \overline{\mathfrak{m}}_{i t}=\phi_{t} \overline{\boldsymbol{m}}_{i t}$. Property 1 and Property 2, imply $\Lambda_{t+1}\left(\mathfrak{a}_{i t+1}\right)=\phi_{t+1} \boldsymbol{m}_{i t+1}+\phi_{t+1}^{1} b_{i t+1}$. Hence, the value $\Delta_{t}$ in (13) can be written as

$$
\max _{\overline{\mathfrak{m}}_{i t} \in \mathfrak{M}_{t}}\left\{-\phi_{t} \overline{\boldsymbol{m}}_{i t}+\max _{\mathfrak{a}_{i t+1}, \mathfrak{n}_{i t+1}^{-}} \beta E_{t} \mathcal{L}\left(\phi_{t+1} \boldsymbol{m}_{i t+1}+\phi_{t+1}^{1} b_{i t+1}\right)\right\},
$$

subject to

$$
\begin{aligned}
\text { s.t. } \quad \mathfrak{n}_{i t+1}^{-}(\mathcal{M})+\mathfrak{m}_{i t+1}(\mathcal{M}) & =\overline{\mathfrak{m}}_{i t}(\mathcal{M}) \quad \text { for all } \mathcal{M} \in \mathcal{F}\left(\overline{\mathcal{M}}_{t}\right) \\
q_{t} b_{i t+1} & =n_{i t+1}^{-}
\end{aligned}
$$

and (9). The constraints (84) and (85) are obtained from (7) and (8) at equality (the objective is increasing in the controls). In (85) I have used $n_{i t+1}^{-} \equiv \mathfrak{n}_{i t+1}^{-}\left(\overline{\mathcal{M}}_{t}\right)$, and the fact that Property 1 implies $q_{t}(s)=q_{t}$ for all $s$, and hence $q_{t} \cdot \mathfrak{b}_{i t+1}=q_{t} b_{i t+1}$. Notice that a solution to (83) is given by any collection measures $\left(\overline{\mathfrak{m}}_{i t}, \mathfrak{m}_{i t+1}, \mathfrak{b}_{i t+1}, \mathfrak{n}_{i t+1}^{-}\right)$that satisfy $\overline{\mathfrak{m}}_{i t} \in \overline{\mathfrak{M}}_{t}$, (84), and (9), as long as the quantities, $\left(\overline{\boldsymbol{m}}_{i t}, \boldsymbol{m}_{i t+1}, b_{i t+1}, n_{i t+1}^{-}\right)$solve

$$
\max _{\overline{\boldsymbol{m}}_{i t}}\left\{-\boldsymbol{\phi}_{t} \overline{\boldsymbol{m}}_{i t}+\max _{\boldsymbol{m}_{i t+1}, b_{i t+1}} \beta E_{t} \mathcal{L}\left(\boldsymbol{\phi}_{t+1} \boldsymbol{m}_{i t+1}+\phi_{t+1}^{1} b_{i t+1}\right)\right\}
$$

subject to $\bar{m}_{i t}^{s}, m_{i t+1}^{s}, b_{i t+1} \in \mathbb{R}^{+}$for $s=0,1$, and $n_{i t+1}^{-}=q_{t} b_{i t+1}$. Since (84) must hold for all $\mathcal{M} \in \mathcal{F}\left(\overline{\mathcal{M}}_{t}\right)$, it holds for $\mathcal{M}=\overline{\mathcal{M}}_{t}^{s}$, which implies $\mathfrak{n}_{i t+1}^{-}\left(\overline{\mathcal{M}}_{t}^{s}\right)+m_{i t+1}^{s}=\bar{m}_{i t}^{s}$ for $s=0,1$. With $n_{i t+1}^{s-} \equiv \mathfrak{n}_{i t+1}^{-}\left(\overline{\mathcal{M}}_{t}^{s}\right)$ and $q_{t} b_{i t+1}^{s} \equiv n_{i t+1}^{s-}$, these constraints can be written as

$$
q_{t} b_{i t+1}^{s}+m_{i t+1}^{s}=\bar{m}_{i t}^{s}, \quad \text { for } s=0,1 .
$$

Thus, a solution to (83) is given by any collection measures $\left(\overline{\mathfrak{m}}_{i t}, \mathfrak{m}_{i t+1}, \mathfrak{b}_{i t+1}, \mathfrak{n}_{i t+1}^{-}\right)$that satisfy $\overline{\mathfrak{m}}_{i t} \in \overline{\mathfrak{M}}_{t}$, (84), and (9), such that the associated quantities $\left(\bar{m}_{i t}^{s}, m_{i t+1}^{s}, b_{i t+1}^{s}, n_{i t+1}^{s-}\right)_{s=0,1}$ solve

$$
\max _{\overline{\boldsymbol{m}}_{i t}}\left\{-\boldsymbol{\phi}_{t} \overline{\boldsymbol{m}}_{i t}+\max _{\boldsymbol{m}_{i t+1}, \boldsymbol{b}_{i t+1}} \beta E_{t} \mathcal{L}\left(\boldsymbol{\phi}_{t+1} \boldsymbol{m}_{i t+1}+\phi_{t+1}^{1} b_{i t+1}\right)\right\},
$$

subject to (86) and $\bar{m}_{i t}^{s}, m_{i t+1}^{s}, b_{i t+1} \in \mathbb{R}^{+}$for $s=0,1$, with $n_{t+1}^{s-}=q_{t} b_{t+1}^{s}$ and $b_{i t+1}=b_{i t+1}^{0}+$ $b_{i t+1}^{1}$. Use (86) to substitute $\boldsymbol{m}_{t+1}$ from the objective. Then a solution to (83) is given by any collection of measures $\left(\overline{\mathfrak{m}}_{i t}, \mathfrak{m}_{i t+1}, \mathfrak{b}_{i t+1}, \mathfrak{n}_{i t+1}^{-}\right)$that satisfy $\overline{\mathfrak{m}}_{i t} \in \overline{\mathfrak{M}}_{t}$, (9), and (84), such that the associated quantities $\left(\bar{m}_{i t}^{s}, m_{i t+1}^{s}, b_{i t+1}^{s}, n_{i t+1}^{s-}\right)_{s=0,1}$ achieve

$$
\Delta_{t}=\max _{\overline{\boldsymbol{m}}_{i t} \in \mathbb{R}^{+} \times \mathbb{R}^{+}}\left\{-\boldsymbol{\phi}_{t} \overline{\boldsymbol{m}}_{i t}+\max _{0 \leq q_{t} \boldsymbol{b}_{i t+1} \leq \overline{\boldsymbol{m}}_{i t}} \beta E_{t} \mathcal{L}\left[\lambda_{t+1}\left(\overline{\boldsymbol{m}}_{i t}, \boldsymbol{b}_{i t+1}\right)\right]\right\},
$$

with $m_{i t+1}^{s}=\bar{m}_{i t}^{s}-q_{t} b_{i t+1}^{s}$ and $n_{i t+1}^{s-}=q_{t} b_{i t+1}^{s}$ for $s=0,1$, and $b_{i t+1}=b_{i t+1}^{0}+b_{i t+1}^{1}$, as stated in the lemma. 


\section{Related literature}

As mentioned in Section 6, for the most part the literature has not attempted to tackle the rate-of-return-dominance puzzle, with the exception of Aiyagari, Wallace, and Wright (1996), and Zhu and Wallace (2007).

Aiyagari, Wallace, and Wright (1996) study a version of Trejos and Wright (1995) or Shi (1995) with two assets: fiat money, and pure discount nominal bond which can be distinguished from money, but has all the same relevant physical characteristics as money. They introduce a positive measure of "government agents" whose role is to issue and redeem bonds. Government agents are just like private agents in every respect, except for the fact that their trading behavior is specified exogenously in three instances. First, when in a single-coincidence meeting with a private seller, a government agent holding a unit of money is programmed to pay with the unit of money with some exogenous probability, or to issue a security and use the newly-issued security as a means of payment, with complementary probability. (In this context, "issuing a security" effectively means physically transforming the unit of money into a bond.) Second, when in a single-coincidence meeting with a private buyer, the government agent refuses to trade with some exogenous positive probability if the buyer carries an unmatured bond. Third, any government agent who meets a private agent who is holding a matured bond, turns the matured bond into a unit of money. This model provides two explanations for the coexistence of money and interest-bearing securities, in the sense that the model has two types of steady states in which such coexistence can occur.

The first rationalization is based on a steady state (which exists for all parametrizations) in which money and matured bonds trade at par, but unmatured bonds are traded at a discount because - by assumption - government agents refuse to accept unmatured bonds in exchange for goods, with positive probability. The second rationalization is based on a steady state (which

only exists for some parametrizations) in which matured bonds trade at a discount. (Since both assets are fiat objects, it is not surprising that there exist steady states with different relative values between them.) In this case, given that private agents regard money as being more valuable than a matured bond, both matured and unmatured bonds will trade at a discount. This discount originates from the fact that in an environment in which it takes an exogenous, random period of time to be able to find a government agent who will redeem a bond, a private agent may end up locked into a matured bond for some time, and hence forced to hold an asset 
that is less valuable than money. In the first rationalization, the discount on bonds is possible by the assumption that government agents discriminate against unmatured bonds. In the second rationalization, it is private agents who endogenously "discriminate" against matured bonds, and this brings about a discount on all bonds. Clearly, for this result to follow, it is necessary to assume that the redemption of matured bonds involves search-type frictions or other imperfections that may prevent a bond holder from redeeming the bond at maturity.

For the last fifteen years, Aiyagari, Wallace and Wright (1996) has been on the frontier of what search-theoretic models of money have to offer in way of explanation of the rate-ofreturn-dominance puzzle. However, because they were working with a pure random-search model, they were forced to adopt a rather contrived bond sales and redemption mechanism. In their formulation, bonds can only be issued as payment for goods in bilateral exchanges (so there is nothing that approximates a real-world open-market auction of government securities), and matured bonds can only get redeemed at random times, whenever the bond holder happens find himself in a bilateral meeting with a government agent. There is a sense in which there is "too much random matching" in the model. This is not just an aesthetic concern; the fact that bond holders are forced to go through a time-consuming period of search for a government agent in order to redeem their bonds is critical for their results. Specifically, if no private agent is locked-in with matured bond holdings that he would like to redeem, then coexistence of money and interest-bearing securities can only be the result of a deliberate government policy to discriminate against its own government bonds by arbitrarily instructing government agents to refuse unmatured bonds in bilateral trades. The moneyspots model I have developed above has several advantages relative to Aiyagari, Wallace and Wright (1996). The most obvious, is that all assets are divisible and there are no restrictions on individual asset holdings, so the model lends itself to standard policy analysis. Also, notice that the coexistence results in Section 4 do not rely on bond redemptions being subject to random-matching frictions, since all agents can (and in fact do) redeem their bond holdings at maturity. ${ }^{29}$

Zhu and Wallace (2007) (see also the related discussion piece, Wallace, 2003) obtain coexistence of money and interest-bearing nominal bonds as an equilibrium, by assuming a bilateral trading protocol that confers larger gains from trade to buyers who hold a larger proportion

\footnotetext{
${ }^{29}$ Without assuming frictions that prevent agents from redeeming bonds when they so desire, and without moneyspots, having nominal bonds and money circulate at different prices is not as easy as constructing an equilibrium in which "blue money" and "red money" circulate at different prices. The reason is that, by standing ready to redeem matured bonds with notes, the government imposes a boundary condition that fixes the relative price of bonds and notes at unity. No such boundary condition exists in the blue-and-red money case.
} 
of money in their portfolios. Specifically, their trading protocol is modeled as a two-step maximization problem. In the first step, buyer utility is maximized subject to a cash-in-advance constraint and to the utility of no trade as a lower bound on seller utility. In the second step, seller utility is maximized subject to the maximum value of utility of the buyer in the first step as a lower bound on buyer utility. There are many allocations (a continuum) in the pairwise core implied by the portfolios of the buyer and seller in the bilateral meeting, which range from giving all the gains from trade to the buyer to giving all the gains from trade to the seller. This feature gives Zhu and Wallace freedom to make a selection from the pairwise core that depends on the composition of the portfolios brought into the meeting (although the compositions of those portfolios are payoff irrelevant). Effectively, the two-step maximization problem selects an allocation from the pairwise core in such a way as to give potential buyers an incentive to bring money to the meetings. The outcome that results from the two-step maximization problem, and hence the ensuing coexistence theory, could be formulated by replacing the maximization problem with a bargaining procedure in which the buyer's bargaining power is an increasing function of his money holdings. In contrast, the protocol used to divide the gains from trade between buyers and sellers in the moneyspot model of Section 4 does not favor money over bonds-buyers get all the gains from trade in every meeting, regardless of the composition of the portfolio that they bring into the meeting. In terms of similarities, the multiplicity of moneyspot equilibria has a parallel in the multiplicity of core allocations in Zhu and Wallace. 


\section{References}

[1] Aiyagari, S. Rao, Neil Wallace, and Randall Wright. "Coexistence of Money and InterestBearing Securities." Journal of Monetary Economics 37(3) (June 1996): 397-419.

[2] Andolfatto, David. "On the Coexistence of Money and Bonds." Simon Fraeser University manuscript, 2005.

[3] Berentsen, Aleksander, and Christopher Waller. "Outside Bonds Versus Inside Bonds." University of Zurich Working Paper 372, 2008.

[4] Bryant, John, and Neil Wallace. "A Suggestion for Further Simplifying the Theory of Money." Federal Reserve Bank of Minneapolis Staff Report 62, 1980.

[5] Cass, David, and Karl Shell. "Do Sunspots Matter?" Journal of Political Economy 91(2) (April 1983): 193-227.

[6] Cecchetti, Stephen G. "The Case of the Negative Nominal Interest Rates: New Estimates of the Term Structure of Interest Rates during the Great Depression." Journal of Political Economy 96(6) (December 1988): 1111-1141.

[7] Galván, Carlos. "Se agrava la escasez de monedas y empiezan a faltar billetes de \$2." Clarín, January 24, 2008.

[8] Gherity, James A. "Interest-bearing Currency: Evidence from the Civil War Experience." Journal of Money, Credit, and Banking 25(1) (February 1993): 125-131.

[9] Grossman, Sanford, and Laurence Weiss. "A Transactions-Based Model of the Monetary Transmission Mechanism." American Economic Review 73(5) (December 1983): 871-880.

[10] Halmos, Paul R. Measure Theory. Springer Verlag, New York, 1974.

[11] Hellwig, Martin F. "The Challenge of Monetary Theory." European Economic Review 37(2) (April 1993): 215-242.

[12] Hicks, J. "A Suggestion for Simplifying the Theory of Money." Economica 9(5) (February 1935): 1-19. 
[13] Jones, Larry E. "Existence of Equilibria with Infinitely Many Consumers and Infinitely Many Commodities." Journal of Mathematical Economics 12(2) (October 1983): 119-138.

[14] Kareken, John, and Neil Wallace. "On the Indeterminacy of Equilibrium Exchange Rates." Quarterly Journal of Economics 96(2) (May 1981): 207-222.

[15] Kocherlakota, Narayana. "Societal Benefits of Illiquid Bonds." Journal of Economic Theory 108(2) (February 2003): 179-193.

[16] Lagos, Ricardo, and Randall Wright. "A Unified Framework for Monetary Theory and Policy Analysis." Journal of Political Economy 113(3) (June 2005): 463-484.

[17] Lucas, Robert E., Jr. "Liquidity and Interest Rates." Journal of Economic Theory 50(2) (April 1990): 237-264.

[18] Makinen, Gail E., and G. Thomas Woodward. "Some Anecdotal Evidence Relating to the Legal Restrictions Theory of the Demand for Money." Journal of Political Economy 94(2) (April 1986): 260-265.

[19] Makinen, Gail E., and G. Thomas Woodward. "Use of Interest-Bearing Currency in the Civil War: The Experience below the Mason-Dixon Line." Journal of Money, Credit, and Banking 31(1) (February 1999): 121-129.

[20] Marchesiani, Alessandro, and Pietro Senesi. "Money and Nominal Bonds." Macroeconomic Dynamics 13(2) (April 2009): 189-199.

[21] Rotemberg, Julio J. "A Monetary Equilibrium Model with Transactions Costs." Journal of Political Economy 92(1) (February 1984): 40-58.

[22] Shi, Shouyong. "Money and Prices: A Model of Search and Bargaining." Journal of Economic Theory 67(2) (December 1995): 467-496.

[23] Shi, Shouyong. "Nominal Bonds and Interest Rates." International Economic Review 46(2) (May 2005): 579-612.

[24] Surowiecki, James. "Change we can't believe in." The New Yorker, June 8, 2009.

[25] Trejos, Alberto, and Randall Wright. "Search, Bargaining, Money, and Prices." Journal of Political Economy 103(1) (February 1995): 118-141. 
[26] U.S. Treasury. Press Release, 2008. http://www.ustreas.gov/offices/domestic-finance/debtmanagement/interest-rate/daily_treas_bill_rates_historical_2008.shtml.

[27] Wallace, Neil. "On Simplifying the Theory of Money." Federal Reserve Bank of Minneapolis Staff Report 22 (June 1977).

[28] Wallace, Neil. "A Legal Restrictions Theory of the Demand for 'Money' and the Role of Monetary Policy." Quarterly Review Federal Reserve Bank of Minneapolis (Winter 1983).

[29] Wallace, Neil. "A Suggestion for Oversimplifying the Theory of Money." Quarterly Review Federal Reserve Bank of Minneapolis (Winter 1990).

[30] Wallace, Neil. "A Dictum for Monetary Theory." Quarterly Review Federal Reserve Bank of Minneapolis (Winter 1998).

[31] Wallace, Neil. "Coexistence of Money and Higher-Return Assets-Again." Penn State University manuscript, 2003.

[32] WuDunn, Sheryl. “Zen Banking: Japan's Negative Interest Rates." The New York Times, November 7, 1998.

[33] Zhu, Tao, and Neil Wallace. "Pairwise Trade and Coexistence of Money and Higher-Return Assets." Journal of Economic Theory 133(1) (March 2007): 524-535. 